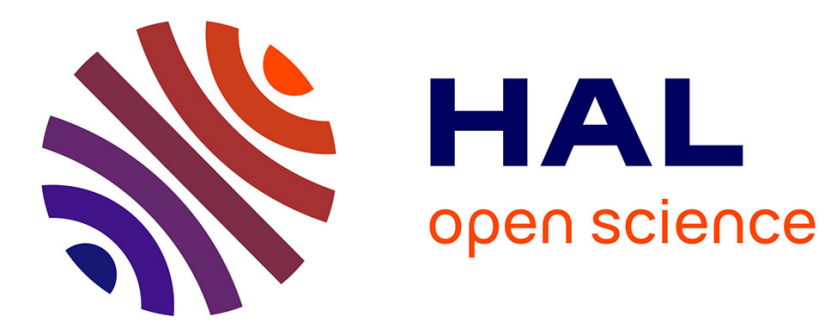

\title{
Modifying module boundaries to design remanufacturable products: the modular grouping explorer tool
}

Nicolas Tchertchian, Dominique Millet, Olivier Pialot

\section{- To cite this version:}

Nicolas Tchertchian, Dominique Millet, Olivier Pialot. Modifying module boundaries to design remanufacturable products: the modular grouping explorer tool. Journal of Engineering Design, 2013, 24 (8), pp.546-574. 10.1080/09544828.2013.776671 . hal-01064187

\section{HAL Id: hal-01064187 \\ https://hal.science/hal-01064187}

Submitted on 17 Sep 2014

HAL is a multi-disciplinary open access archive for the deposit and dissemination of scientific research documents, whether they are published or not. The documents may come from teaching and research institutions in France or abroad, or from public or private research centers.
L'archive ouverte pluridisciplinaire HAL, est destinée au dépôt et à la diffusion de documents scientifiques de niveau recherche, publiés ou non, émanant des établissements d'enseignement et de recherche français ou étrangers, des laboratoires publics ou privés. 


\title{
Modifying module boundaries to design remanufacturable products: the modular grouping explorer tool
}

\author{
Nicolas Tchertchian, Dominique Millet, Olivier Pialot
}

Ecodesign and Optimization of Product,LISMMA, Supmeca, Toulon, France

Provide full correspondence details here including e-mail for the corresponding author

Provide short biographical notes on all contributors here if the journal requires them.

Sommaire

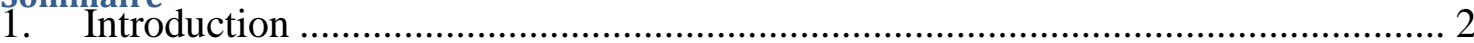

2. Etat de l'art des méthodes d'optimisation de la fin de vie des produits .................. 3

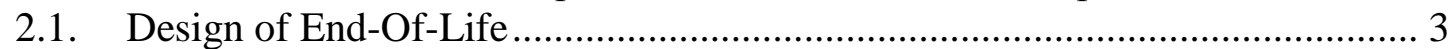

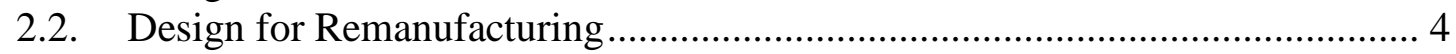

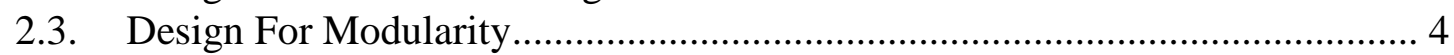

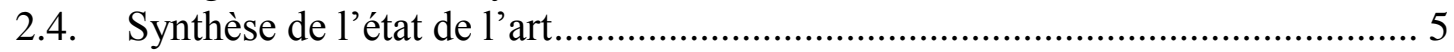

3. Présentation théorique de l'outil « Modular Grouping Explorer ».......................... 6

3.1. Les 3 principes fondateurs de l'outil MGE.................................................... 7

3.1.1. Modification de l'architecture : Nouvelle définition des limites des
modules/ composants.............................................................................................. 7

3.1.2. Caractérisation des modules initiaux ....................................................... 7

3.1.3. Indicateur de reconfiguration basé sur des affinités (critéres de groupement d'éclatement de modules/ composants) ................................................. 8

3.2. Les 4 étapes de la méthode MGE (Logique globale de la méthode) ................ 8

3.2.1. Phase 1 : Définition de la classe de chaque module ................................. 8

3.2.2. Phase $2:$ Validation de la remanufacturabilité des modules ....................... 9

3.2.3. Phase 3 : Réorganisation des modules d'après les critères de fiabilité, d'obsolescence et de gamme ............................................................................... 10

3.2.4. Phase 4 : Evaluation Economique et Environnementale de l'architecture produit 11

3.3. Génération des Architectures Produit ............................................................ 12

4. Modular Grouping Explorer tool appliqué à une machine Expresso ...................... 14

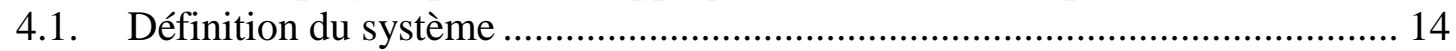

4.2. Etude A : Evaluation Architectures produits potentielles............................... 16

4.3. Etude B : Influence des « Targets » environnemental et économique.............. 18

4.4. Etude $\mathrm{C}$ : Sensibilité sur les valeurs MTBF et VLT ….................................... 19

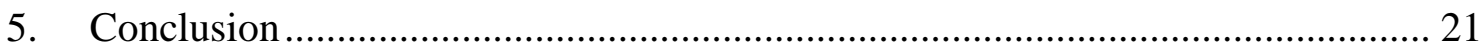

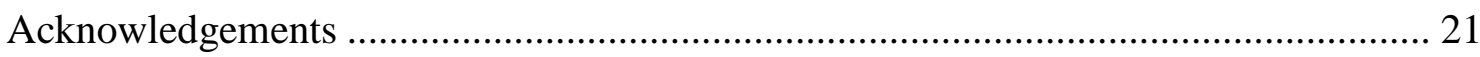

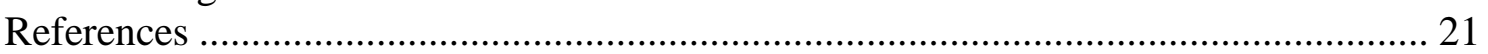




\title{
How to modify modules boundaries to design remanufacturable Product? The Modular Grouping Explorer tool
}

\begin{abstract}
Un des challenges de cette décennie est de rationaliser la consommation de toutes nos ressources (matières et énergie) en privilégiant une économie soutenable. Le remanufacturing est une des solutions pour atteindre ces objectifs; cependant cette option de fin de vie doit être optimisée. Les approches proposées actuellement dans la littérature visent soit à identifier la meilleure option de fin de vie pour une architecture de produits donnée soit à optimiser la séquence de désassemblage au moyen d'algorithme soit à regrouper des composants en fonction des options de fin de vie. Aucune méthode explicite ne propose une redéfinition complète de l'architecture pour l'adapter à des fin de vie durable, c'est-à-dire en augmentant la proportion de modules remanufacturables et recyclables. La méthode proposée dans ce papier permet une identification a priori des modules remanufacturables et recyclables à partir des critères de coûts et d'impact environnemental, des regroupements entre les modules remanufacturables ou des modules recyclables à partir des critères de fiabilité et d'obsolescence, et une redéfinition des périmètres des modules. L'approche proposée est testée sur une étude de cas (espresso machine) ; cette étude met en exergue les avantages d'une telle approche (une exploration exhaustive des architectures produit tout en permettant de redéfinir les frontières des modules) ainsi que ses limites.
\end{abstract}

Keywords: Lifecycle design; Modular design; remanufacturing; Eco-design tool

\section{Introduction}

Les problèmes environnementaux provoqués par l'émergence de nouveaux marchés et du développement rapide de la consommation de biens nécessitent une rationalisation de la consommation des ressources mondiales tout en maintenant une croissance de l'activité industrielle locale. Le remanufacturing est un moyen d'y parvenir en restaurant un produit usagé (en fin de vie) dans un état fonctionnel équivalent à un produit neuf. (Lund 1996, Ijomah 2002).

Cette définition contraint à intégrer dans la conception de produits remanufacturables les contraintes induites par les opérations relatives au remanufacturing définies par Steinhilper (1998) ou Sundin (2004) tel que le désassemblage, le nettoyage, l'inspection, le tri, le reconditionnement et le réassemblage. La modularisation du produit est une voie pour permettre l'optimisation des opérations liées à la fin de vie du produit comme la réutilisabilité, le recyclage, la maintenabilité et la mise à jour technologique (Umeda 2008). Dans la littérature, la modularisation des produits s'insère dans les approches DfX. Design for Modularity est une approche visant à subdiviser un système en parties plus petites qui peuvent être créés de manière indépendante et ensuite utilisées dans différents systèmes. Outre la réduction des coûts, la modularité offre de la flexibilité dans la conception (l'ajout de nouvelles solutions techniques). La conception modulaire est par ailleurs utile d'un point de vue industriel car elle permet une différentiation retardée des produits dans la ligne de production en réduisant la complexité de production.

Les approches d'écoconception proposées actuellement dans la littérature pour optimiser les impacts environnementaux induits par la fin de vie d'un produit visent essentiellement à identifier la meilleure option de fin de vie pour une architecture de produits donnée au dépend d'une redéfinition complète de l'architecture permettant d'augmenter la proportion de modules remanufacturables et recyclables.

Pour répondre à ce manque, cet article est structuré en 6 sections. Dans la section 2, nous effectuons un état de l'art dans le domaine du Design for end-of-life, design for remanufacturing et du design for modularity. Dans la section 3, nous définissons tout d'abord les 3 principes fondateurs de l'outil proposé : (1) remise en cause des frontières des modules, (2) prédéfinition des modules remanufacturables et recyclables initiaux et (3) reconfiguration basées sur des calculs d'affinité. Ensuite, nous décrivons de façon théorique chaque étape de l'outil MGE. Dans la section 4, l'outil MGE est expérimenté sur un cas spécifique (la machine à expresso) qui donne lieu à trois analyses particulières. Enfin la section 5 discute les résultats de ces trois analyses et conclut cette étude en positionnant l'outil à l'intérieur d'une méthodologie de conception plus globale. 


\section{Etat de l'art des méthodes d'optimisation de la fin de vie des produits}

De nombreuses méthodes ont été développées dans la littérature depuis les années 80 . Nous verrons dans un premier temps les méthodes qui aident à identifier la meilleure stratégie de fin de vie de produits (recyclage, réutilisation, remanufacturing). Dans un deuxième temps, nous ferons le bilan des méthodes de type Design for Remanufacturing. Enfin nous terminons ce chapitre par une analyse des méthodes Design for modularity.

\subsection{Design of End-Of-Life}

Les réglementations européennes WEEE (EU, 2003) et ELV (EU, 2000) exigent que les entreprises valorisent leurs produits en fin de vie en réponse aux préoccupations sociétales sur la diminution des ressources, l'occupation des sols, les impacts sur la santé et l'environnement provoqués par le traitement des produits contenant des éléments nocifs (par exemple les produits électroniques).

Beaucoup d'entreprises traitent les problèmes liés à la fin de vie de leurs produits à la fin du processus de conception lorsque la plupart des paramètres sont figées conduisant souvent à des modifications locales, non pertinentes sur le plan global. Rose (1998) définit la stratégie de fin de vie comme une étape à part entière de la définition du produit. Un des facteurs de réussite d'une meilleure conception pour l'environnement est de planifier la stratégie de fin de vie le plus en amont possible dans le processus de conception (Ishii 1992) avant même de considérer les détails techniques imposés par le recyclage ou remanufactring (Rose et al. 1998). Dans ce contexte, Tonnelier et al. (2005) propose une méthode d'évaluation qualitative de l'aptitude au recyclage (RPE) de pièces automobiles intégrée en amont du processus de conception chez le constructeur automobile PSA. L'évaluation du traitement d'un véhicule à la fin de sa vie implique deux familles des critères : les critères liés aux matériaux (number of materials, compatibility, separability, etc.), et ceux liés au potentiel de démontage (accessibility, standardization of fixings, modularity of components, etc.).

De nombreux outils et méthodes ont été développés par les chercheurs à destination des concepteurs, mais ils sont rarement mis en pratique [Rose et al. 2000] ; ils nécessitent en effet des connaissances avancées ainsi qu'une expertise bien établie sur le traitement des déchets en fin de vie. Or le niveau d'intégration de ces problématiques dans l'entreprise n'est pas toujours suffisant. Rose et al. (1999) soulignent "Design for recyclability requires knowledge, rather than assumptions, about how recyclers will treat various subassemblies, components, and materials to recover residual value".

La plupart des méthodes existantes cherchent à optimiser la fin de vie en identifiant la meilleure stratégie de traitement ; le remanufacturing, la réutilisation, le recyclage (primaire et secondaire), l'incinération (avec ou sans récupération d'énergie) et la mise en décharge afin de réduire à la fois les impacts et les coûts (Rose et al. 1998, 2000, 2002), (Zwolinski et al. 2008).

Ces méthodes analysent les invariants ou les tendances d'une grande diversité de produits existants afin de déterminer quelles sont les stratégies les mieux adaptées pour un produit. Au milieu des années 90, la caractérisation de facteurs ou critères de réussite de la fin de vie a fait l'objet de nombreux travaux. Parmi ces travaux citons ceux de Rose (2000) et de son équipe, qui ont évalué 37 produits, l'analyse du cycle de vie de ces produits (dont la fin de vie constitue des exemples de bonne pratique et de succès) a permis une classification en fonction de leur fin de vie ( Remanufacturing, réutilisation, recyclage et fin de vie).

L'analyse de tous ces produits a permis à Rose de les caractériser à l'aide de facteurs internes et externes puis de les catégoriser en groupe ou typologie de produits selon la valeur de ces facteurs.

Rose (2000) considère les 6 caractéristiques de produits suivantes: usure, obsolescence, niveau d'intégration, nombre de composants, cycle de conception et raisons de re-conception comme ayant une influence majeure sur la sélection (prédiction) de la stratégie de fin de vie. Ces caractéristiques sont ensuite utilisées pour prédire la stratégie de fin de vie des produits en conception à l'aide d'un arbre de décision par un outil d'analyse de groupement (CART).

Xing et al. (2003) adopte une démarche similaire en se basant sur la caractérisation des produits selon les 4 facteurs suivant : usure, obsolescence, niveau d'intégration fonctionnel et raisons de re-conception.

Cela conduit généralement à privilégier l'option remanufacturing qui requiert une durée de vie et un cycle technologique plus long. La définition de ces caractéristiques doit tenir compte de la facilité d'accès de l'information dans les premières phases de la conception comme facteur de réussite à propager les lignes directrices (Rose et al. 1998). Romery et al. (2011) propose une méthode d'évaluation de scénario de fin de vie (ELSEM) permettant la caractérisation de la fin de vie des modules selon 6 options de fin de vie dès les premières phases de la conception. L'évaluation est basée sur 15 paramètres influents dont la durabilité, la valeur du module, la quantité de de matériaux à haute valeur, la difficulté de désassemblage, la quantité de matériaux différents, la quantité de modules, etc. 


\subsection{Design for Remanufacturing}

L'origine du Remanufacturing remonte à la deuxième guerre mondiale pendant laquelle de nombreux produits utilisés ont été remanufacturés de façon artisanale afin de maintenir la société en état de marche. Ce n'est qu'à la fin des années 90 que le concept de remanufacturing, comme processus industriel maitrisé, se développe dans les domaines industriels et académiques avec des travaux fondateurs (Lund 1996), (Steinhilper 1998). La définition du remanufacturing (Ijomah 2002) implique plusieurs domaines de la conception dont l'évaluation de la remanufacturabilité consistant à identifier les facteurs de réussites du remanufacturing (Williams et Shu, 2001), (Sundin, 2001), (Zwolinski et al. 2008), la reconception de produit pour faciliter le processus de remanufacturing (Van der Laan and Salomon 1997), (Ijomah et al. 2007), (Duflou et al. 2008), la recherche sur les opérations nécessaires à la remise en état de produit (désassemblage, nettoyage, inspection et tri, reconditionnement et réassemblage) (Lund 1996), (Steinhilper 1998), les évaluations environnementale (Gehin 2008) et économique (Kerr 1999), (Mont et al. 2006). La plupart de ces publications ne traite souvent qu'un seul aspect de ce champ très vaste. Par exemple les travaux de recherche de (Gungor and Gupta 1997), (Lambert 2002), (Desai and Mital 2003) portent sur les moyens à mettre en place pour optimiser le désassemblage des produits en vue de leur valorisation. La complexité croît en effet avec le nombre de composants et le temps requis pour le désassemblage dépend de nombreux paramètres (interconnexion des composants entre eux, types de fixation, direction de désassemblage, etc.) En 1997 Gungor and Gupta (1997) proposèrent une méthode heuristique pour définir la séquence de désassemblage optimale mais sans tenir compte des aspects environnementaux et économiques. Takeuchi et al. (2006) ont développé une méthode d'optimisation multi-objectif utilisant les algorithmes génétiques pour réduire le temps de désassemblage avec comme fonctions objectifs le coût et l'impact environnemental (consommation énergétique en MJ). Dans toutes ces publications, le désassemblage complet d'un produit le plus rapidement possible est difficilement justifiable du point de vue économique (Lee 2001). En fait le remanufacturing doit considérer plusieurs critères car, comme Kara et al. (2006) le soulignent: «full disassembly of product tends to be unproductive due to technical and cost constraint». Zuidwijk and Krikke (2007) insistent: « a product recovery strategy determines the degree of disassembly of a product and the assignment of recovery options. Le produit peut être considérablement amélioré si l'architecture est conçue de telle manière que les premières actions du démontage libèrent les pièces les plus sensibles au remanufacturing (Giudice, 2008). Lambert (2002) introduit le concept de désassemblage incomplet justifié par certaines contraintes techniques : connections irréversibles, contraintes économiques (puisque les coûts de désassemblage sont inversement proportionnels au profit généré par la réutilisation des composants extraits).

\subsection{Design For Modularity}

Tseng et al. (2009) soulignent la complexité du problème de remanufacturabilité : "because end-of-life strategies for the entire products are not homogenous, it's more meaningful to determine appropriate endof-life strategy in terms of sustainability for constituting components or subassemblies of the entire product...". Ils recommandent de spécifier les stratégies de fin de vie en fonction des composants et de sous-ensembles. Pour y remédier, Xing et al. (2006) puis Wang and Tseng (2010) suggèrent de regrouper les composants ayant des propriétés communes au sein d'un même module. C'est ainsi que les méthodes de type «Design for Modularity» ont émergé pour réaliser un désassemblage intelligent et profitable. La modularisation des produits semble en effet un premier pas vers une conception durable (Seliger and Zettl 2008). Les produits modulaires permettent d'améliorer la valorisation des matériaux en différenciant les modules qui sont potentiellement recyclables de ceux qui ne le sont pas (Kimura et al. 2001). Il semble ainsi que la conception des produits futurs devra mieux prendre en compte la définition des modules et de l'architecture produit. La majorité des produits sur le marché, aujourd'hui, n'ont pas de module clairement défini du point de vue de la fin de vie, compliquant ainsi l'évaluation des scénarios de fin de vie. Dans les produits ayant un grand nombre de composants, des sous-ensembles peuvent être définis correspondant aux groupes fonctionnels du produit, comme le cas d'étude sur la machine à laver décrit par Kara et al. (2006). Le remanufacturing permet ainsi de réutiliser des produits ou des modules sur plusieurs cycles selon les exigences du consommateur ou l'évolution du marché (Seliger and Zettl 2008). Tomiyama et al. (2005) proposent le « Post Mass Production Paradigm» pour réduire la consommation des ressources naturelles ainsi que la production de déchets. Ce paradigme vise une augmentation de la durée de vie des modules en introduisant le concept de l'obsolescence fonctionnelle du produit. "Longerlife products should have functional upgradability besides reliability and fault-tolerance" (Kondoh et al. 1998) (Umeda et al. 2008). Un aspect peu discuté dans la littérature concerne les bénéfices de l'adoption d'une approche modulaire dans le cycle de vie du produit. Le produit n'est plus le résultat d'un assemblage de matériaux et d'un processus de fabrication mais une somme de modules interconnectés jouant le rôle d'unité centrale dans la construction du modèle (Tchertchian et al. 2009). Gehin (2008) a développé ainsi un modèle dans lequel le produit est composé de modules suivant des stratégies de 
valorisation sur plusieurs cycles d'utilisation. Son approche permet une évaluation environnementale du produit à partir de ces modules mais sans considérer les coûts opérationnels qu'impose une réutilisation sur plusieurs cycles (supply chain, refurbishing...). Kimura et al. (2001) et Umeda et al. (2008) proposent des méthodes de conception modulaire basée à la fois sur les caractéristiques du cycle de vie et des informations géométriques. Selon eux, les composants qui subissent le même cycle de vie devraient être regroupés au sein d'un même module. Ces modules peuvent alors subir une maintenance, un remanufacturing ou un processus de recyclage sans nécessiter un démontage complet du produit. Cela conduit à une gestion plus aisée des composants tout au long du cycle de vie et à la réduction de l'impact environnemental et des coûts de logistique et de récupération.

Wang and Tseng (2010) propose ainsi des regroupements modulaires basés sur les options de fin de vie des composants du produit : des regroupements des modules remanufacturables, des regroupements des modules réutilisables, des regroupements des modules recyclables. Pour aider le concepteur à choisir la meilleure architecture de produit, cet auteur introduit un indice de commonalité de cycle de vie (Life Cycle Commonality Metrics noté LCCM). Le principe est de calculer la proportion de composants dans un module subissant la même stratégie de fin de vie. L'indice LCCM à une valeur comprise entre 0 et 1 , les architectures les plus homogènes sont celles qui ont des modules composés de composant subissant la même fin de vie avec un indice LCCM égal à 1.

Xing et al. (2006) introduisent des indicateurs de similarité afin de voir si deux composants ne peuvent pas être groupés en un même module. Ils définissent 3 types de similarité :

- Similarité de cycle technologique: c'est-à-dire le degré de ressemblance de la longévité technologique

- Similarité de détérioration physique : c'est-à-dire le degré de ressemblance de la durée de vie

- Similarité d'exigence de service : c'est-à-dire le degré de ressemblance des opérations de service et de maintenance

L'agrégation de chaque coefficient de similarité permet de définir un indicateur de compatibilité de cycle de vie (Life Cycle Commonality).

\subsection{Synthèse de l'état de l'art}

L'état de l'art présenté dans les paragraphes précédents, met en lumière différents niveaux dans la remise cause du produit, des frontières des modules et des composants et de la structure des produits.

Il y a les méthodes qui permettent de définir une stratégie de fin de vie appropriée en comparant les caractéristiques du produit à optimiser avec les différentes typologies de produits. Néanmoins, afin de faire correspondre au mieux le produit à optimiser avec une typologie de produits il peut être nécessaire de procéder à des modifications. Cette approche conduit le plus souvent à envisager des modifications mineures en s'appuyant sur des règles de conception (guidelines). Par exemple, Xing et al. (2006) listent 10 règles de conception pour la sélection des matériaux, par exemple : éviter l'utilisation de matériaux toxiques, favoriser l'emploi de matières recyclées et recyclables, minimiser la variété des matériaux, utiliser des matériaux compatibles, choisir des matériaux compatibles avec la stratégie de fin de vie... et 10 règles de conception pour la sélection de fixations, par exemple : standardiser les fixations, minimiser le nombre de type de fixations, limiter l'utilisation de fixation irréversible (soudure, rivets...), choisir des fixations compatibles avec les matériaux des composants... 
Table 1: Comparaison d'outils et méthodes pour l'optimisation de la stratégie de fin de vie de produits

\begin{tabular}{|c|c|c|c|c|c|}
\hline & $\begin{array}{l}\text { Rose }(1998,2000) \\
\text { ELDA }\end{array}$ & $\begin{array}{l}\text { Lee (2001) } \\
\text { Guidelines }\end{array}$ & $\begin{array}{l}\text { Takeuchi (2006) } \\
\text { Genetic algorithms }\end{array}$ & $\begin{array}{l}\text { Kiritsis (2003) } \\
\text { AEOLOS }\end{array}$ & $\begin{array}{l}\text { Xing (2003) } \\
\text { IREDA }\end{array}$ \\
\hline Characterisation & $\begin{array}{l}\text { Number of } \\
\text { functions } \\
\text { Technological } \\
\text { cycle } \\
\text { Level of } \\
\text { Integration } \\
\text { Level of } \\
\text { cleanliness } \\
\text { Number of } \\
\text { materials } \\
\text { Number of parts }\end{array}$ & $\begin{array}{l}\text { Guidelines on } \\
\text { material } \\
\text { specification }\end{array}$ & $\begin{array}{l}\text { Components' } \\
\text { characteristics } \\
\text { Liaisons } \\
\text { Environmental } \\
\text { impact } \\
\text { End-of-Life } \\
\text { legislation } \\
\text { Infrastructure cost } \\
\text { Business cost }\end{array}$ & $\begin{array}{l}\text { Technical Life } \\
\text { Economic Life } \\
\text { Social Life } \\
\text { Legal Life }\end{array}$ & $\begin{array}{l}\text { Lifetime } \\
\text { Technological } \\
\text { cycle } \\
\text { Level of } \\
\text { Integration } \\
\text { Reason of redesign }\end{array}$ \\
\hline Option & $\begin{array}{l}\text { Remanufacturing } \\
\text { Primary recycling } \\
\text { Secondary } \\
\text { recycling } \\
\text { Disposal }\end{array}$ & $\begin{array}{l}\text { Landfill } \\
\text { Primary recycling } \\
\text { Secondary } \\
\text { recycling } \\
\text { Incineration } \\
\text { (Energy recovery) } \\
\text { Reuse/ } \\
\text { Remanufacturing } \\
\end{array}$ & $\begin{array}{l}\text { Recycling } \\
\text { Reuse } \\
\text { Landfill }\end{array}$ & $\begin{array}{l}\text { Remanufacturing } \\
\text { Recycling } \\
\text { Incineration with } \\
\text { energy recovery } \\
\text { Incineration } \\
\text { without energy } \\
\text { recovery } \\
\text { Landfill } \\
\end{array}$ & $\begin{array}{l}\text { Reuse } \\
\text { Remanufacturing } \\
\text { Recovery }\end{array}$ \\
\hline $\begin{array}{l}\text { Level of } \\
\text { modification }\end{array}$ & $\begin{array}{l}\text { End-of-Life } \\
\text { strategy } \\
\text { optimization }\end{array}$ & $\begin{array}{l}\text { Minor } \\
\text { modification of } \\
\text { product } \\
\text { architecture based } \\
\text { on guidelines }\end{array}$ & $\begin{array}{l}\text { Optimisation } \\
\text { based on genetic } \\
\text { algorithm. } \\
\text { Long process of } \\
\text { generation of the } \\
\text { architecture. } \\
\begin{array}{l}\text { Number } \\
\text { solutions to be } \\
\text { handled not } \\
\text { controlled not }\end{array}\end{array}$ & $\begin{array}{l}\text { End-of-Life } \\
\text { strategy } \\
\text { optimization }\end{array}$ & $\begin{array}{l}\text { Minor } \\
\text { modification of } \\
\text { product } \\
\text { architecture based } \\
\text { on guidelines }\end{array}$ \\
\hline
\end{tabular}

Ces listes de règles ne modifient pas radicalement l'architecture du produit; elles permettent principalement d'optimiser les coûts liés aux opérations de désassemblage et d'améliorer l'efficacité du recyclage.

D'autres règles de conception engendrent des modifications de l'architecture du produit de façon à améliorer les opérations de fin de vie du produit. Ces règles reposent sur une caractérisation des composants en termes d'impacts sur l'environnement et coûts/ ou gains économiques. Lee et al. (2001) proposent des règles de positionnement des modules dans l'architecture du produit en fonction de ces deux critères (économique et environnemental), par exemple : positionner les composants qui engendrent le plus de coûts de démontage en bas de l'architecture (destination décharge sans démontage), positionner les composants les moins impactant au sommet de l'architecture, positionner les composants les plus impactant en bas de l'architecture, sauf si un traitement particulier est nécessaire dans ce cas il sera positionner au premier niveau du graphe de démontage, simplifier les éléments de fixation pour réduire le temps de désassemblage des composants et utiliser des composants à fortes valeurs économiques et ou à faibles impacts environnementaux.

Afin de concevoir des produits moins impactants, nous proposons une alternative aux méthodes existantes (Table 1) se positionnant entre :

1. les approches par optimisation de la stratégie de fin de vie (qui, en explorant incomplètement l'espace des solutions, conduisent à des modifications superficielles) et,

2. les approches par reconfiguration complète du produit - modules et composants- (qui, en envisageant des modifications à tous les niveaux du produit -modules, composants, fixations-, conduisent à une explosion combinatoire des solutions à évaluer). systématiques longues où le concepteur n'a pas la maîtrise des solutions générées (Figure 1).

En autorisant une redéfinition des frontières de certains modules correctement choisis ainsi qu'en modifiant certains composants ciblés, notre approche conduit potentiellement à une exploration suffisante du champ du possible tout en minimisant le travail du groupe de conception impliqué.

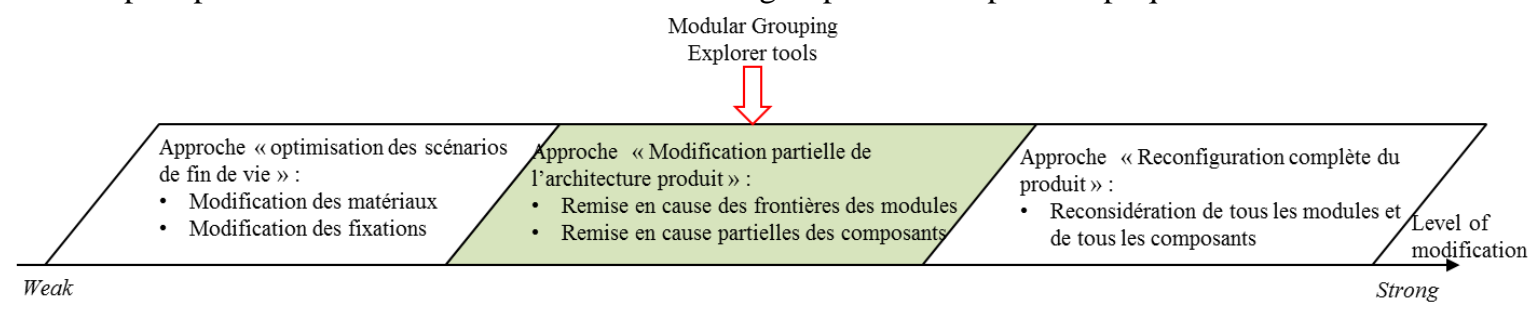

Figure 1 : Positionnement original de notre approche 'Modular Grouping Explorer'

\section{Présentation théorique de l'outil « Modular Grouping Explorer »}

Dans cette section nous présenterons d'abord les 3 principes fondateurs puis les 4 étapes de la méthode. 


\subsection{Les 3 principes fondateurs de l'outil MGE}

Les 3 principes fondateurs de l'outil MGE concernent : (1) la remise en cause des frontières des modules, (2) la prédéfinition de la nature remanufacturable ou recyclable des modules initiaux, et (3) le regroupement de modules par un calcul d'affinité.

\subsubsection{Modification de l'architecture : Nouvelle définition des limites des modules/ composants}

L'architecture produit peut être définie comme la somme de composants plus ou moins ordonnés en modules qui réalisent une ou plusieurs fonctions (Ulrich 1995). Chacun de ces composants sont liés par des interfaces (liaisons physiques) nécessaires à la cohésion du produit (Erens and Verhulst, 1997), (Fixon, 2005).

Par convention, nous définissons l'architecture produit de la façon suivante :

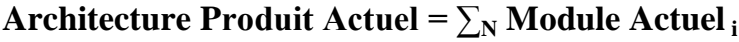

Avec Module actuel $_{\mathbf{i}}=\sum_{\mathbf{L}}$ Composant $_{\mathbf{j}}$

L'interface entre composants est le plus souvent optimisée pour faciliter les opérations d'assemblage ou de service après-vente actuellement. Dans la plupart des produits un module (association de composants) peut être formé d'un ensemble de composants hétérogènes dans leur traitement de fin de vie (cf. Figure 2.a).

L'objectif premier de l'outil est d'aider le concepteur à modifier la structure du produit en déformant (splitter ou grouper) les modules et en changeant l'organisation des modules à l'intérieur du produit.

L'architecture produit que nous proposons est définie de la façon suivante :

Nouvelle Architecture Produit $=\sum_{\mathbf{N}}$, Nouveau Module $_{1}$

$=\sum_{M}$ Module Actuel $_{\mathbf{i}}-\sum_{\mathbf{P}}$ Composant $_{\mathbf{j}}+\sum_{\mathbf{Q}}$ Nouveau Composant $_{\mathbf{k}}$

Les modules sont ainsi homogénéisés en fonction de la similarité de la fin de vie des composants. (Cf. Figure 2.b). La réduction des coûts et du temps de désassemblage passent par une optimisation de la séquence de désassemblage. Les modules à haute valeur ajoutée nécessitent une extraction facile (sans dommages) et rapide. Pour cela les modules doivent, lorsque c'est possible, être situés au sommet de la séquence de démontage. Au contraire les modules destinés au recyclage, à l'incinération ou à la mise en décharge peuvent être situés à la fin de la séquence de démontage Lee et al. (2001).

\subsubsection{Caractérisation des modules initiaux}

La caractérisation des modules initiaux repose sur un algorithme permettant une caractérisation des modules en pMR (c'est-à-dire Module potentiellement Remanufacturable), en pMr (c'est-à-dire Module potentiellement recyclable) et en modules intermédiaires pMU (c'est-à-dire module potentiellement Undefined qui caractérisent des modules remanufacturables et/ou recyclables). L'enjeu est de décomposer ou regrouper ces modules pMU pour les transformer soit en pMR soit en pMr.

Afin de classer chaque module identifié, les critères environnementaux et économiques ont été retenus. Ils permettent en effet de rendre compte, (qualitativement), des vraies préoccupations dans l'industrie à savoir : (1) diminuer les impacts environnementaux (pour respecter les réglementations de plus en plus sévères) et (2) augmenter les profits. La caractérisation des modules se fait en fonction de la répartition des coûts et des impacts du produit. Le prix de revient a été utilisé pour caractériser le coût économique du module, tandis que l'impact environnemental est caractérisé par l'impact de la phase extractionfabrication du module (sans tenir compte des phases d'utilisation, distribution et fin de vie) ; ce choix méthodologique (focalisation sur la Pre-life et non sur le cycle de vie) permet d'une part de simplifier le problème et d'autre part d'identifier objectivement les plus forts gisements économicoenvironnementaux. 
a) Current Product Architecture

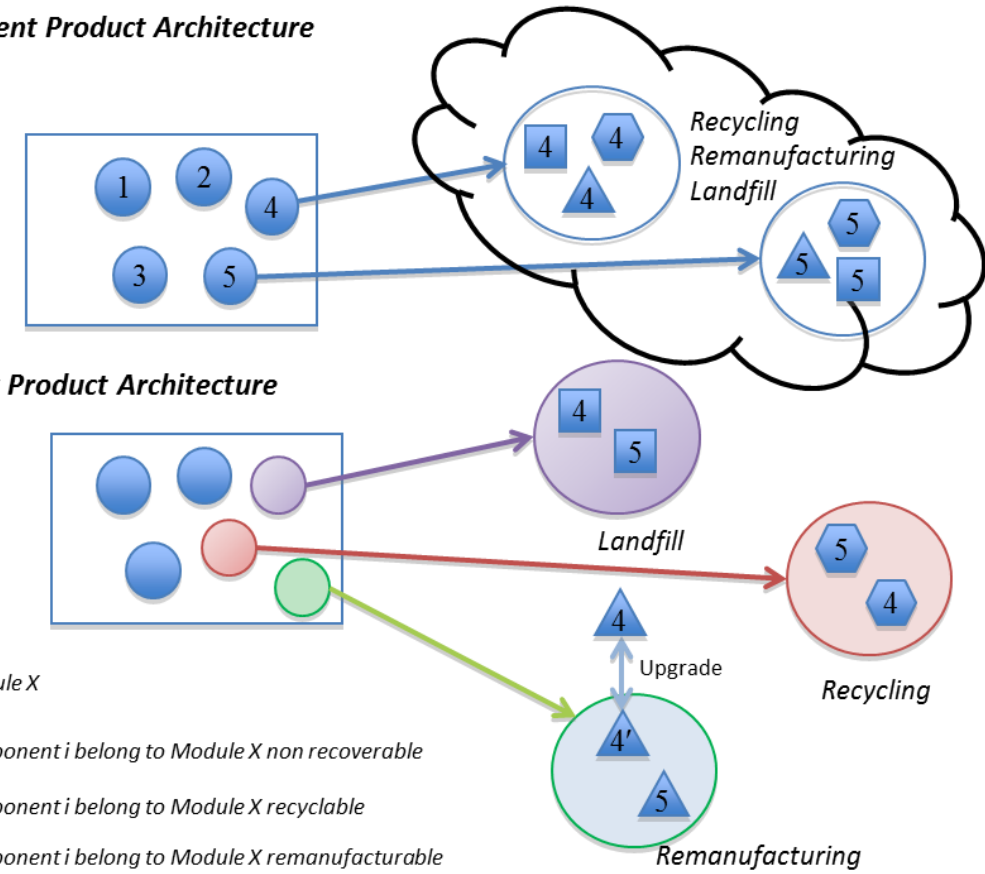

Figure 2 : Définition des Architectures Produit

\subsubsection{Indicateur de reconfiguration basé sur des affinités (critéres de groupement d'éclatement de modules/ composants)}

D'après la littérature pour améliorer l'architecture modulaire d'un produit il est nécessaire de regrouper les modules (ou composants) en fonction de différents critères.

"Considering a whole life cycle of a product, components that undergo the same life cycle processes should be grouped into one module for improving, e.g., disassemblability, maintainability, upgradability, reusability, and recyclability" (Umeda et al. 2008).

Deux types de regroupement peuvent être envisagés :

- $\quad$ Regroupement entre modules recyclables pMr

- $\quad$ Regroupement entre modules remanufacturables pMR

Pour ce faire, nous nous appuyons sur la notion d'affinité qui permet de rendre compte d'un potentiel de regroupement entre deux modules (ou sous-modules) selon qu'ils partagent certaines propriétés. Nous avons retenu trois propriétés principales permettant de regrouper des modules du point de vue de la fin de vie:

- L’homogénéité des matériaux

- La fiabilité/ maintenabilité

- L'obsolescence technique et visuelle

\subsection{Les 4 étapes de la méthode MGE (Logique globale de la méthode)}

La méthode MGE est composée de quatre phases développées dans les paragraphes suivants et synthétisées par le logigramme Figure 6.

\subsection{1. $\quad$ Phase 1 : Définition de la classe de chaque module}

Dans un premier temps les modules du produit sont caractérisés en fonction de leurs propriétés économiques et environnementales.

- Evaluation environnementale : L'impact environnemental (ei) pris en compte pour évaluer les modules correspond ici à l'impact environnemental généré lors des phases d'Extraction et de Production.

- Evaluation économique : Le coût de production (pc) pris en compte est le prix de revient des modules.

Le fait de caractériser un module potentiellement remanufacturable par de forts coûts et de forts impacts environnementaux traduit la volonté de diminuer les coûts en réutilisant des modules à fortes valeurs ajoutées et de réduire les impacts en évitant l'extraction de matériaux très impactants pour la fabrication de nouveaux modules. La méthode retenue pour classer les modules selon une option de fin de vie est 
qualitative; les seuils de classement dans les catégories fort/faible sur le plan économique ou environnemental doivent être définis collégialement de façon itérative (Table 2).

Trois types de modules sont définis :

Table 2 : Caractérisation des modules.

\begin{tabular}{|c|c|c|c|c|}
\hline Characteristics & $\begin{array}{c}\text { High cost/ } \\
\text { High impact }\end{array}$ & $\begin{array}{c}\text { Low cost/ } \\
\text { Low impact }\end{array}$ & $\begin{array}{c}\text { High cost / } \\
\text { Low impact }\end{array}$ & $\begin{array}{c}\text { Low cost / } \\
\text { High impact }\end{array}$ \\
\hline Conditions & $\begin{array}{c}\mathrm{pc}>\mathrm{T}^{\mathrm{pc}} \\
\mathrm{ei}>\mathrm{T}^{\mathrm{ei}}\end{array}$ & $\begin{array}{c}\mathrm{pc}<\mathrm{T}^{\mathrm{pc}} \\
\mathrm{ei}<\mathrm{T}^{\mathrm{ei}}\end{array}$ & $\begin{array}{c}\mathrm{pc}>\mathrm{T}^{\mathrm{pc}} \\
\mathrm{ei}<\mathrm{T}^{\mathrm{ei}}\end{array}$ & $\begin{array}{c}\mathrm{pc}<\mathrm{T}^{\mathrm{pc}} \\
\mathrm{ei}>\mathrm{T}^{\mathrm{ei}}\end{array}$ \\
\hline $\begin{array}{c}\text { Potential Modules }(\mathrm{pMs}) \\
\text { Categories }\end{array}$ & $\mathrm{pMR}$ & $\mathrm{pMr}$ & $\mathrm{pMU}$ & $\mathrm{pMU}$ \\
\hline
\end{tabular}

L'équipe multidisciplinaire doit définir les seuils : (1) le seuil «production cost » $\mathrm{T}^{\mathrm{pc}}$ : Fort coût/Faible coût (en pourcentage du prix de revient total du produit) et (2) le seuil « environmental impact » $\mathrm{T}^{\mathrm{ei}}$ : Fort impact/Faible impact environnemental (en pourcentage de l'impact environnemental à l'extractionfabrication du produit). L'impact environnemental pourra être évalué selon une catégorie d'impact particulière (par exemple GWP en kg eq CO2) ou bien par un indicateur de type «end-score » (éco-points de la méthode EI 99 par exemple). L'équipe de conception définit n valeurs « seuil » (exemple en Figure 3) correspondant à différents seuils Fort coût/Faible coût et Fort impact/Faible Impact. En élargissant le champ des possibilités, le risque d'erreur de définition de seuil (Fort/Faible) est réduit. Les listes de modules obtenues reflètent une stratégie particulière de l'équipe : focalisée (sur des modules très coûteux et très impactants) ou non focalisée (en considérant une liste plus large de modules moins coûteux et moins impactants).
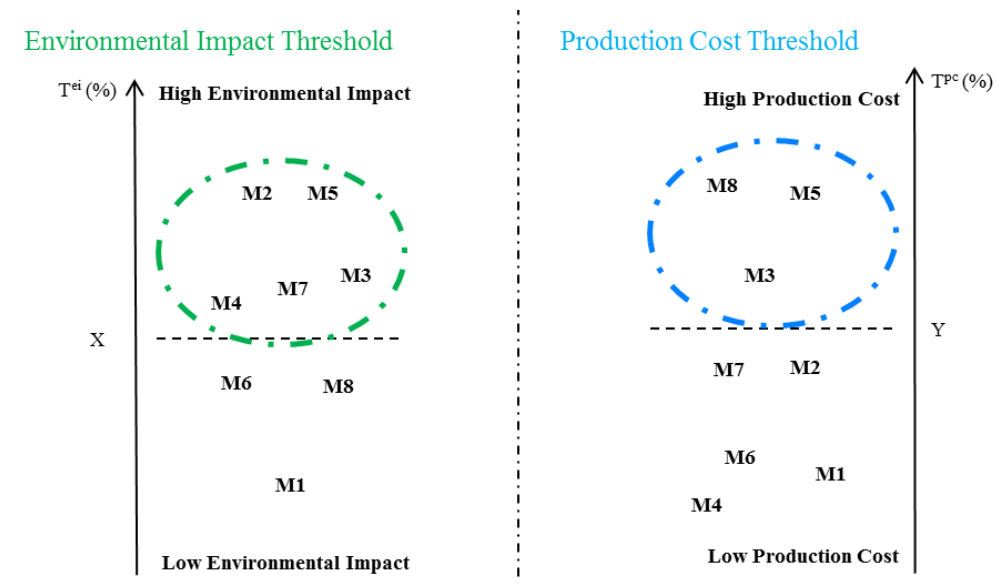

Figure 3 : Identification des modules à haut impact environnemental - ou haut prix de revient

Cette première phase permet ainsi de classer les modules en trois grandes familles de modules potentiels (pMs) : pMR, pMr et pMU. Selon l'exemple de la Figure 3, en considérant les seuils Tei $=\mathrm{X}$ et Tpc $=\mathrm{Y}$ les modules M3 et M5 sont des pMR car leur impact environnemental $>\mathrm{X}$ et leur coût de production $>\mathrm{Y}$. Suivant ces seuils les modules M1 et M6 sont des pMr car leur impact environnemental $<\mathrm{X}$ et leur coût de production < Y. Les modules M2, M4 et M7 sont indéfinis pMU car leur impact environnemental $>\mathrm{X}$ mais leur coût de production $<\mathrm{Y}$, tandis que M8 est pMU car son impact environnemental $<\mathrm{X}$ et son coût de production $>\mathrm{Y}$.

\subsubsection{Phase 2 : Validation de la remanufacturabilité des modules}

La deuxième phase est une étape de contrôle qui permet de vérifier la remanufacturabilité des modules pMR (Figure 4).

Le contrôle se fait en utilisant deux filtres :

- un filtre Fiabilité : Le module remanufacturé doit être apte à supporter plusieurs cycles d'utilisation sans tomber en panne. La vérification de la fiabilité se fait à l'aide du calcul du MTBF : « Mean Time Between Failure ».

$\operatorname{MTBF}(\mathrm{pMR})>\mathrm{NC} \times \mathrm{DC}$.

Avec $\mathrm{NC}=$ Number of Cycles of product

et $\mathrm{DC}=$ Duration of Cycles of product 
- Un filtre Obsolescence : Le module remanufacturé doit toujours être au gout du jour technologiquement et visuellement pour l'utilisateur d'un produit remanufacturé. L'obsolescence est déterminée qualitativement par la durée VLT : «Value Life Time » (Umeda et al, 2008).

$\mathrm{VLT}(\mathrm{pMR})>\mathrm{NC} \times \mathrm{DC}$.

Les aspects suivants doivent ensuite être vérifiés :

- Vérification de l'obsolescence : En cas de forte obsolescence l'équipe de conception doit envisager l'option d'Upgrade/Update (pMR+). Si un upgrade n'est pas envisageable alors ce module doit être reclassé pMr.

- Vérification de la fiabilité : En cas de faible fiabilité l'équipe de conception doit envisager l'option de Reconception avec augmentation de la fiabilité ; si cela est réalisable, alors le module est classé pMR. Si l'augmentation de la fiabilité du module n'est pas envisageable, deux options se présentent : soit décomposer le module en deux modules $\mathrm{pMr}+\mathrm{pMR}$, soit définir le module comme pMr.

- En cas de faible obsolescence et forte fiabilité le module sera défini comme pMR.

- Si un module pMR n'est pas suffisamment fiable ou s'il est rapidement obsolète alors il sera redéfini comme pMr.

\begin{tabular}{|c|c|c|c|c|c|}
\hline \multirow{2}{*}{$\begin{array}{c}\text { End-of-Life } \\
\text { Option }\end{array}$} & \multicolumn{2}{|c|}{ Characterisation } & \multicolumn{2}{|c|}{ Validation } & End-of-Life \\
\cline { 4 - 6 } & Cost & Impact & Reliability & Obsolescence & Option checked \\
\hline \multirow{3}{*}{$\mathrm{pMR}$} & \multirow{3}{*}{+} & & + & + & $\mathrm{pMR}$ \\
\cline { 4 - 6 } & & + & - & - & $\mathrm{pMr}$ \\
\cline { 4 - 6 } & & & + & - & $\mathrm{pMR}$ \\
\cline { 4 - 6 } & & & - & + & $\mathrm{pMR}+\mathrm{pMr}$ \\
\hline
\end{tabular}

Figure 4 : Validation de la caractérisation des Modules pMR

\subsubsection{Phase 3 : Réorganisation des modules d'après les critères de fiabilité, d'obsolescence et de gamme}

Cette étape permet de regrouper les principaux modules du produit en assemblage de modules ou de sousmodules potentiellement remanufacturables ou recyclables. Les sous-modules sont issus de la décomposition des modules pMU (en sous-modules homogènes $\mathrm{R}$ ou $\mathrm{r}$ ) dans la phase 2 . Le processus de regroupement des modules est basé sur le calcul d'un coefficient d'affinité entre modules (pMR ou pMr) à partir de l'équation Affinity $=\alpha$.Afiab $+\beta$. Aobs $+\gamma$. Acom (1) (Table 3), entre sous modules ou entre modules et sous modules. L'affinité peut se définir comme un potentiel d'interaction entre deux modules (ou sous modules) en fonction de certains critères.

Cette affinité comporte 3 composantes: affinité de fiabilité, affinité d'obsolescence et affinité de commonalité. La finalité de cette affinité est de privilégier les regroupements entre des modules dont les coefficients sont les plus forts. L'état de l'art ainsi que des applications menées sur un cas industriel permettent de privilégier trois critères [Umeda et al., 2008], [Tseng and Chang, 2008]. La décomposition de l'affinité en plusieurs composantes à deux points forts :

- Un score agrégé permet une évaluation et une interprétation des résultats mieux adaptées à une utilisation de l'outil par une équipe multidisciplinaire.

- L'autre avantage est la possibilité de rajouter des coefficients de pondération aux critères, permettant ainsi de mieux prendre en considération des stratégies particulières (focalisée sur des exigences utilisateurs, concepteurs ou pour véhiculer une caractéristique de l'entreprise, par exemple : favoriser le regroupement des composants les moins fiables pour simplifier le remplacement du module). 
Avec

- $\quad \mathrm{A}_{\text {rel }}$ : Composante fiabilité :

Données d'entrée : MTBF composants Modules existants.

$A_{r e l}=1-\frac{\operatorname{Max}\left(M T B F_{p M X i}, M T B F_{p M X_{j}}\right)-\operatorname{Min}\left(M T B F_{p M X i}, M T B F_{p M X_{j}}\right)}{\operatorname{Max}\left(M T B F_{p M X i}, M T B F_{p M X_{j}}\right)}$

- $\quad \mathrm{A}_{\mathrm{obs}}$ : Composante obsolescence :

Calcul de la composante obsolescence du module $\mathrm{pMY}_{\mathrm{j}}$ avec le module $\mathrm{pMX}_{\mathrm{i}}$

Evaluation qualitative en fonction de l'évolution des technologies et des usages des consommateurs.

$$
A_{o b s}=1-\frac{\operatorname{Max}\left(V L T_{p M X i}, V L T_{p M X j}\right)-\operatorname{Min}\left(V L T_{p M X i}, V L T_{p M X j}\right)}{\operatorname{Max}\left(V L T_{p M X i}, V L T_{p M X j}\right)}
$$

- $\mathrm{A}_{\text {com }}$ : Composante Commonalité :

Données d'entrée : Gamme produit/ Modules dans chaque gamme. Soit i et j, les deux modules dont on souhaite calculer leur affinité par rapport à la commonalité et soit $\mathrm{p}$, le nombre de produits pris en compte dans la gamme.

$$
A_{\text {com }}=\frac{\sum_{k=1}^{p} \delta_{i j}^{k}}{p}
$$

If $\mathrm{i}$ and $\mathrm{j}=\mathrm{k}$ then $\delta=1$ else $\delta=0$

Table 3 : Calcul des affinités

Enfin les regroupements pMR et pMr sont validés par un contrôle permettant de s'assurer de la compatibilité fonctionnelle de tous les modules entre eux (flux de matières, d'énergies et d'informations). Ce contrôle peut être réalisé par le biais d'un bloc diagramme fonctionnel (analyse fonctionnelle).

Lorsqu'une incompatibilité est observée, le regroupement n'est pas validé. Il est nécessaire de procéder à une itération.

Pour que l'algorithme soit complet il est laissé une marge de liberté pour que l'équipe de conception puisse modifier les sous-modules (en optimisant les matériaux, ou les fixations...) et les rendre compatibles avec les pMR ou pMr.

\subsubsection{Phase 4 : Evaluation Economique et Environnementale de l'architecture produit}

Les phases amont de l'outil permettent au concepteur d'avoir un large spectre de modules, en fonction de la sensibilité choisie pour les critères : fiabilité, obsolescence et commonalité. Dans la dernière phase le concepteur est assisté dans son choix d'une ou plusieurs architectures. Le choix des architectures dépend de deux critères. Le premier est économique, il prend en considération le coût des opérations de démontage par rapport au prix de revient des modules (ou des composants constitutifs). Le second critère de sélection est environnemental : le choix des architectures prend en compte l'impact des modules sur les phases d'extraction et de fabrication. Le concepteur établit, pour chaque liste de modules, différentes organisations à l'aide de bloc diagramme fonctionnel précisant les flux d'information entre modules. L'objectif est de représenter la séquence de démontage simplement afin de permettre l'extraction en priorité des modules pMR sans démonter les modules pMr. Pour calculer le temps de démontage les informations suivantes sont nécessaires : type de connexions, orientation de démontage, accessibilité, etc. Le temps de démontage est estimé numériquement par un logiciel dédié.

Le coût de désassemblage est donné par l'équation suivante :

$$
C_{d i s}=\sum_{i=1}^{n} t_{p M R i} \times C_{l}
$$

Avec $\mathrm{C}_{\mathrm{dis}}$ : disassembly cost

$\mathrm{t}_{\mathrm{pMRi}}:$ temps de désassemble du module pMRi

Et $\quad \mathrm{C}_{1}$ : coût horaire du travail (Hourly Labour Cost?) 
Pour le critère environnemental, le concepteur calcule les impacts générés durant la phase d'extraction des matériaux et de fabrication des modules. Les modules pMR sont définis par un impact dit «évité » et les modules pMr génèrent des impacts. Les solutions sont classées en fonction des gains environnementaux obtenus.

\subsection{Génération des Architectures Produit}

L'application des 4 phases (Figure 5) de la méthode permet d'explorer le maximum d'Architecture Produit (PA) en fonction d'une stratégie reflétée par le choix des seuils Tei/ Tpc et des coefficients utilisés dans l'affinité $\alpha, \beta$ et $\gamma$.

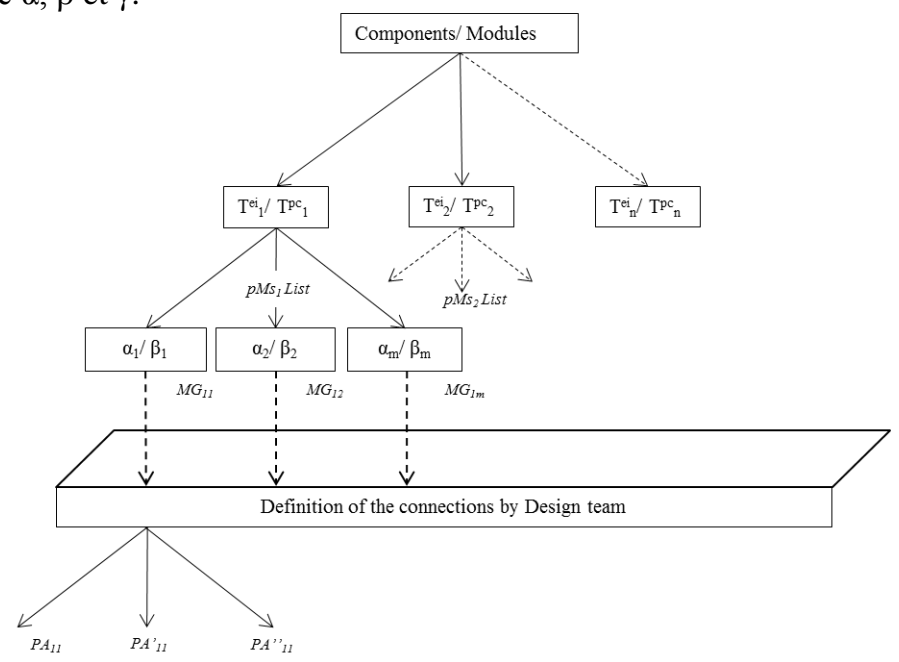

Figure 5 : Processus de génération des Groupements modulaires (MGs) et des Architectures Produit (PA)

Les groupements modulaires (MGs) sont générés par l'outil MGE en fonction des stratégies défendues par l'utilisateur.

En fonction des modules obtenus le concepteurs valide ou invalide le ou les groupements non réalistes. Pour chaque groupement réaliste, le concepteur doit définir les liaisons entre chaque nouveau module pour obtenir une architecture produit (PA) potentielle.

Chaque architecture est évaluée environnementalement (impact évité pour la réutilisation de matériaux et de module et impacts créés pour la fabrication de nouveaux modules et par la fabrication de nouveaux modules et par les processus liés au remanufacturing).

L'outil MGE intègre une phase d'évaluation à la suite du processus d'exploration.

L'évaluation environnementale (phase extraction fabrication) est réalisée à partir du logiciel d'analyse de cycle de vie Simapro et de la base de données Ecoinvent. L'évaluation économique résulte d'une analyse entre module fabriqué, module réutilisé, matériaux recyclés et coût de désassemblage. Pour le calcul des coûts de désassemblage la base de données intégrée au logiciel prodtect est utilisée. 


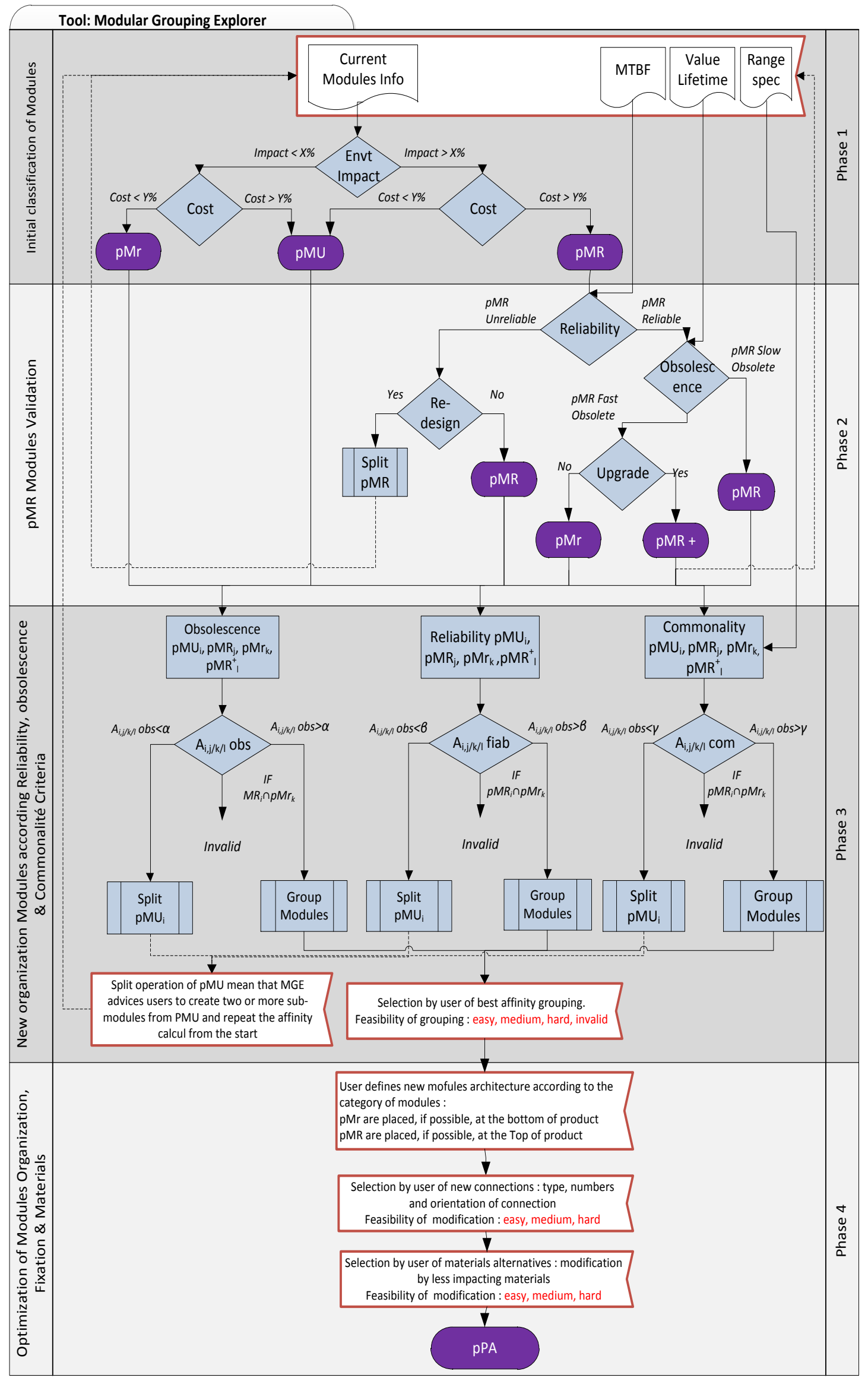

Figure 6 : Logigramme de la méthode. 


\section{Modular Grouping Explorer tool appliqué à une machine Expresso}

Dans cette section, MGE est appliqué à une machine à café à dosette du commerce. En 4.1, une description du système est donnée. Dans les 3 paragraphes suivants, 3 études expérimentales sont présentées :

Etude A: Utilisation de l'outil MGE pour générer des groupements modulaires et explorer les architectures découlant de l'association de ces Groupements Modulaires = Modular Groupings (MGs). Les Critères de coûts et d'impacts sont utilisés comme leviers d'amélioration des performances environnementale et économique selon 3 stratégies : optimisation de la fiabilité (MTBF), optimisation de l'obsolescence (VLT) et une stratégie neutre.

Etude B : Influence des «Seuils » environnemental et économique. Les Seuils sont les pourcentages de prix (ou d'impacts) à partir desquels un module est catégorisé comme coûteux (ou respectivement comme impactant du point de vue environnemental)

Etude C : Etude de sensibilité sur les valeurs MTBF et VLT. Les valeurs de fiabilité et d'obsolescence sont des données qui ne sont pas forcément connues avec précision dans les premières phases de conception de produits.

Dans la suite de cette étude, pour simplifier la compréhension de l'étude de cas, nous considérerons $\gamma=0$.

\subsection{Définition du système}

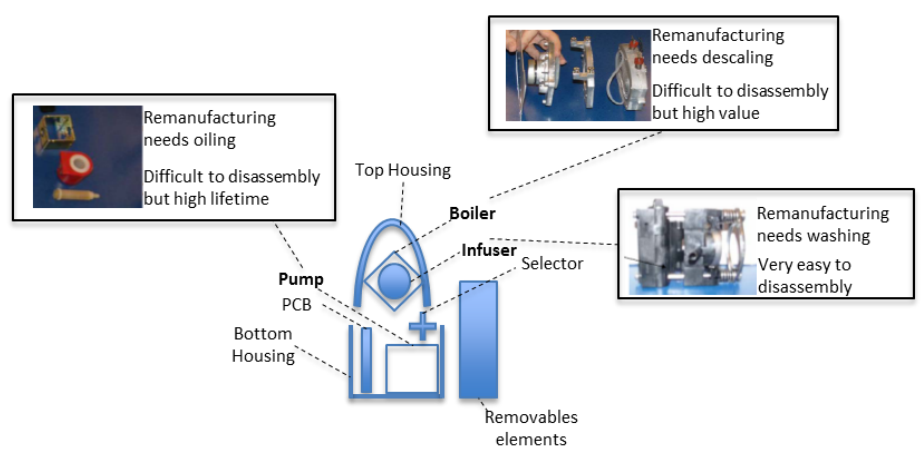

Figure 7. Modules characteristics

La machine à café étudiée a été prédécoupée en 10 modules (Figure 8). Ces 10 modules sont identifiés selon des critères fonctionnels, de liaisons, de dépendances...

Les données fournies en entrée de l'outil MGE sont :

- La dénomination des modules

- Le prix de revient des modules

- L'impact environnemental (en phases d'extraction et de fabrication) des modules (exprimé en éco-points, méthode EI99)

- La fiabilité des modules (MTBF)

- La fiabilité maximale pouvant être atteinte $(\mathrm{MTBF}+)$

- La durée avant obsolescence (VLT)

- La durée avant obsolescence (VLT+) maximale pouvant être atteinte 


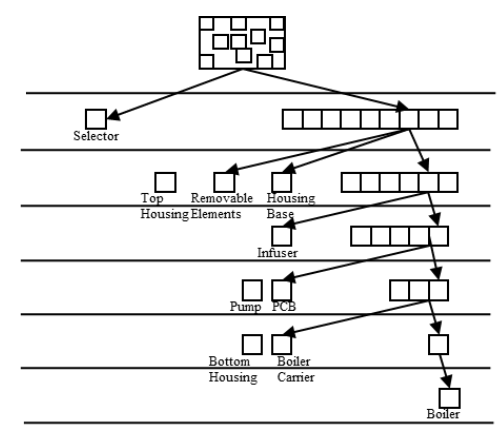

Figure 8 : Dismantling sequence for current product

L'interface d'entrée des données (Figure 9) se présente sous la forme d'un tableur dans lequel l'utilisateur vient remplir et mettre à jour les données de chaque module.

\begin{tabular}{|c|c|c|c|c|c|c|c|c|}
\hline $\mathrm{N}^{\circ}$ & Modules' listing & $\begin{array}{c}\text { Environmental } \\
\text { Impact } \\
\text { Impact (mPt) }\end{array}$ & $\begin{array}{c}\text { Production Cost } \\
\text { Cost }(€)\end{array}$ & MTBF & MTBF & VLT & VLT+ & $\begin{array}{c}\text { Disassembly } \\
\text { Level }\end{array}$ \\
\hline M1 & Selector & 5 & 0,5 & 10 & 10 & 10 & 10 & 1 \\
M2 & Top Housing & 180 & 5 & 9 & 10 & 2 & 5 & 2 \\
M3 & Recoverable Elements & 320 & 22 & 9 & 10 & 6 & 8 & 2 \\
M4 & Housing base & 160 & 4 & 8 & 10 & 2 & 5 & 2 \\
M5 & Infuser & 110 & 16 & 6 & 8 & 5 & 8 & 3 \\
M6 & Pump & 658 & 16,5 & 8 & 15 & 10 & 10 & 4 \\
M7 & PCB & 920 & 3,5 & 5 & 6 & 6 & 8 & 4 \\
M8 & Boiler Carrier & 93 & 3,5 & 10 & 10 & 10 & 10 & 5 \\
M9 & Bottom Housing & 280 & 7 & 10 & 10 & 10 & 10 & 5 \\
M10 & Boiler & 990 & 9 & 2 & 3 & 4 & 5 & 6 \\
\hline
\end{tabular}

Figure 9: Modular Grouping Explorer input data interface

La frontière entre un module coûteux et un module peu coûteux est déterminée par l'utilisateur. Cette frontière se traduit par 2 coefficients indépendant coût/ impact, l'utilisateur peut ainsi privilégier un des deux critères en fonction de la stratégie souhaitée.

Table 4 : tableau de synthèse des regroupements modulaires possibles

\begin{tabular}{|c|c|c|c|}
\hline & Feasibility & Modification/Action & Validation by Design team \\
\hline \multicolumn{4}{|l|}{ pMr Grouping } \\
\hline Selector-Top Housing & Yes & & Yes \\
\hline Selector-Housing Base & Yes & & No \\
\hline Selector-Infuser & No & Split & \\
\hline Selector-PCB & No & Split & \\
\hline Selector-Boiler Carrier & Yes & & No \\
\hline Selector-Boiler & No & No & \\
\hline Top Housing-Housing Base & Yes & & Yes \\
\hline Top Housing-Infuser & No & Split & \\
\hline Top Housing-PCB & No & Split & \\
\hline Top Housing-Boiler Carrier & No & No & \\
\hline Top Housing-Boiler & No & No & \\
\hline Housing Base-Infuser & Yes & Improve reliability infuser & No \\
\hline Housing Base-PCB & No & Split & \\
\hline Housing Base-Boiler Carrier & Yes & & Yes \\
\hline Housing Base-Boiler & No & No & \\
\hline Infuser-Boiler Carrier & No & Split & \\
\hline Infuser-Boiler & No & Split & \\
\hline PCB-Boiler Carrier & No & Split & \\
\hline PCB-Boiler & No & Split & \\
\hline Boiler Carrier-Boiler & Yes & Improve reliability Boiler & Yes \\
\hline \multicolumn{4}{|l|}{ pMR Grouping } \\
\hline Removable Elements-Infuser & No & Split & \\
\hline Removable Elements-Pump & Yes & & No \\
\hline $\begin{array}{l}\text { Removable Elements-PCB } \\
\text { Removable Elements-Bottom }\end{array}$ & No & Split & \\
\hline Housing & Yes & & Possible \\
\hline Infuser-Pump & Yes & Improve reliability Infuser & Yes \\
\hline Infuser-Bottom Housing & No & Split & \\
\hline Pump-PCB & No & Split & \\
\hline Pump-Bottom Housing & Yes & & Yes \\
\hline PCB-Bottom Housing & No & Split & \\
\hline
\end{tabular}




\subsection{Etude A : Evaluation Architectures produits potentielles}

Dans un premier temps, MGE permet à l'utilisateur de catégoriser chaque composant/ module fonctionnel en module potentiellement remanufacturable ( $\mathrm{pMR}$ ), potentiellement recyclable $(\mathrm{pMr})$ ou potentiellement indéfini (pMU) en fonction des seuils de faible/fort impact environnemental et de faible/fort prix de revient. Dans un deuxième temps, après validation, MGE établit des groupements $\mathrm{pMR} / \mathrm{pMR}, \mathrm{pMr} / \mathrm{pMr}$, $\mathrm{pMU} / \mathrm{pMR}, \mathrm{pMU} / \mathrm{pMr}$ par affinité selon les composantes fiabilité et/ ou obsolescence. Pour le cas d'étude, les modules de même classe et ayant une affinité supérieure ou égale à $80 \%$ sont regroupés au sein d'un même module. C'est ensuite l'intelligence du concepteur qui permet de valider ou non les groupements selon les compatibilités techniques et fonctionnelles. Pour les modules dont l'affinité est inférieure aux $80 \%$, l'outil MGE calcule une affinité potentielle basée sur les données d'entrée $\mathrm{MTBF}^{+}$et $\mathrm{VLT}^{+}$(correspondant à l'utilisation d'autres composants plus robuste et/ou moins soumis à l'obsolescence). Dans ce cas, le concepteur est informé dans le tableau de synthèse par une information supplémentaire de type « Améliorer fiabilité du Module x ».

Finalement quand un couple $\mathrm{pMU} / \mathrm{pMX}$ ou $\mathrm{pMX} / \mathrm{pMX}$ (avec $\mathrm{X}=\mathrm{R}$ ou $\mathrm{r}$ ) ne remplit pas tous les critères, l'outil suggère de procéder à une itération en décomposant les modules pMU et/ou pMX en sous-modules lorsque cela est possible (la consigne «SPLIT » indique l'action de décomposition des modules en sous modules/ composants).

Dans l'étude de cas A, les composants/modules sont d'abord catégorisés selon 3 leviers d'amélioration : économique, environnemental et économico-environnemental matérialisés par 3 couples (Tei, Tpc). Il en résulte 3 ensembles de caractérisation de modules. Chacun de ces ensembles est ensuite soumis à 3 stratégies de groupement par affinité : fiabilité, obsolescence et fiabilité/ obsolescence. Il en résulte les 9 groupements modulaires présentés dans la Figure 10 (Les modules libellés en vert correspondent à des pMR et les noirs à des pMr). Par exemple, le premier module (M'1) du groupement modulaire 7 (MG7) regroupe le sélecteur (M1) et le carter supérieur (M2).

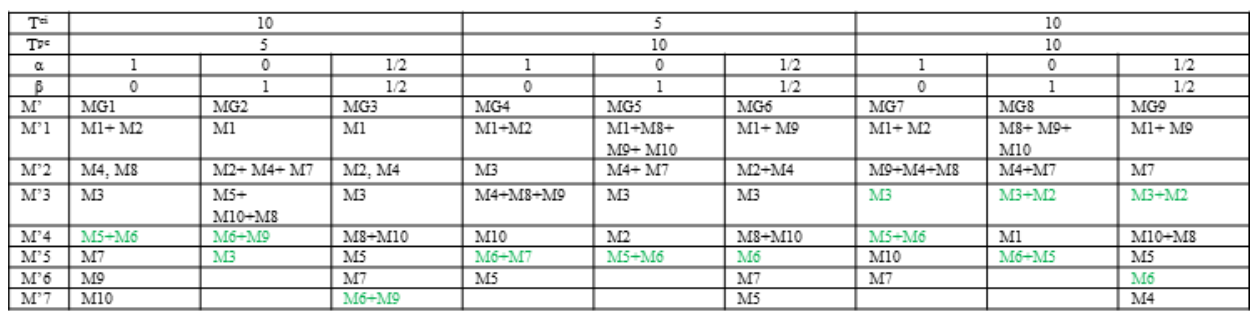

Figure 10: Potential solutions of Product Architecture defined by MGE

Pour chacun des 9 Groupements Modulaires (MG), l'équipe de conception conçoit ensuite les liaisons entre les modules (M') en privilégiant des techniques de fixation facilitant le désassemblage des modules remanufacturables et le recyclage des autres modules. La méthodologie telle qu'elle a été implémentée dans l'outil MGE guide le concepteur dans la construction d'une séquence de désassemblage favorisant le désassemblage des modules remanufacturables.

\begin{tabular}{|c|c|c|c|c|c|c|c|c|c|}
\hline Level/PA & $\mathrm{PA}_{1}$ & $\mathrm{PA}_{2}$ & $\mathrm{PA}_{3}$ & $\mathrm{PA}_{4}$ & $\mathrm{PA}_{5}$ & $\mathrm{PA}_{6}$ & $\mathrm{PA}_{7}$ & $\mathrm{PA}_{8}$ & $\mathrm{PA}_{0}$ \\
\hline 1 & $M^{\prime} 1, M^{\prime} 3$ & $M^{\prime} 1, M^{\prime} 5$ & $M^{\prime} 1, M^{\prime} 3, M^{\prime} 5$ & $\begin{array}{l}\text { M'1, M'2, } \\
\text { M'6 }^{\prime}\end{array}$ & $\begin{array}{l}\text { M'1, M'3, } \\
\text { M'4 }\end{array}$ & $\begin{array}{l}M^{\prime} 1, M^{\prime} 3, \\
M^{2} 7\end{array}$ & $M^{\prime} 1, M^{\prime} 3$ & $\begin{array}{l}M^{2} 2, M^{\prime} 3, \\
M^{\prime} 4\end{array}$ & $\begin{array}{l}M^{\prime} 1, M^{\prime} 3, \\
M^{\prime} 5, M^{7} 7\end{array}$ \\
\hline 2 & $M^{\prime} 4, M^{\prime} 5$ & $\mathrm{M}^{\prime} 4, \mathrm{M}^{\prime} 2$ & $\mathrm{M}^{\prime} 2, \mathrm{M}^{\prime} 6, \mathrm{M}^{\prime} 7$ & $M^{\prime} 3$ & $M^{\prime} 5, M^{\prime} 2$ & $\begin{array}{l}M^{\prime} 5, M^{\prime} 6, \\
M^{\prime} 2\end{array}$ & M'2 & $\begin{array}{l}M^{\prime} 5 \\
\end{array}$ & $\mathrm{M}^{\prime} 4$ \\
\hline 3 & M'7 & M'3 & M'4 & $\mathrm{M}^{\prime} 4, \mathrm{M}^{\prime} 5$ & & M'4 & $\begin{array}{l}M^{2} 4, M^{\prime} 5, \\
M^{\prime} 6\end{array}$ & M'1 & $\mathrm{M}^{\prime} 2, \mathrm{M}^{\prime} 6$ \\
\hline 4 & $\mathrm{M}^{\prime} 6, \mathrm{M}^{\prime} 2$ & & & & & & & & \\
\hline $\mathrm{pMR}$ & M'4 & $M^{\prime} 4, M^{\prime} 5$ & M'7 & M'5 & $M^{\prime} 5$ & M'5 & M'4 & $M^{\prime} 3, M^{\prime} 5$ & $M^{\prime} 3, M^{\prime} 6$ \\
\hline $\mathrm{pMr}$ & $\begin{array}{l}M^{\prime} 1, \\
M^{\prime} 2, \\
M^{\prime} 3, \\
M^{\prime} 5 \\
M^{\prime} 6 \\
M^{\prime} 7\end{array}$ & $\begin{array}{l}M^{\prime} 1, \\
M^{\prime} 2, \\
M^{\prime} 3, \\
M^{\prime} 6, \\
M^{\prime} 7\end{array}$ & $\begin{array}{l}M^{\prime} 1, \\
M^{\prime} 2, \\
M^{\prime} 3 \\
M^{\prime} 4, \\
M^{\prime} 5 \\
M^{\prime} 6\end{array}$ & $\begin{array}{l}M^{\prime} 1, \\
M^{\prime} 2, \\
M^{\prime} 3, \\
M^{\prime} 6, \\
M^{\prime} 7\end{array}$ & $\begin{array}{l}M^{\prime} 1, \\
M^{\prime} 2, \\
M^{\prime} 3, \\
M^{\prime} 4, \\
M^{\prime} 6, \\
M^{\prime} 7\end{array}$ & $\begin{array}{l}\mathrm{M}^{\prime} 1, \\
\mathrm{M}^{\prime} 2, \\
\mathrm{M}^{\prime} 3, \\
\mathrm{M}^{\prime} 4, \\
\mathrm{M}^{\prime} 6, \\
\mathrm{M}^{\prime} 7\end{array}$ & $\begin{array}{l}M^{\prime} 1, \\
M^{\prime} 2, \\
M^{\prime} 3, \\
M^{\prime} 5, \\
M^{\prime} 6, \\
M^{\prime} 7\end{array}$ & $\begin{array}{l}M^{\prime} 1, \\
M^{\prime} 2, \\
M^{\prime} 4, \\
M^{\prime} 6 \\
M^{\prime} 7\end{array}$ & $\begin{array}{l}M^{\prime} 1, \\
M^{2} 2, \\
M^{2} 4, \\
M^{2} 5 \\
M^{\prime} 7\end{array}$ \\
\hline
\end{tabular}

Figure $11: 9$ potential Product Architectures (one for each distinguished MG) 


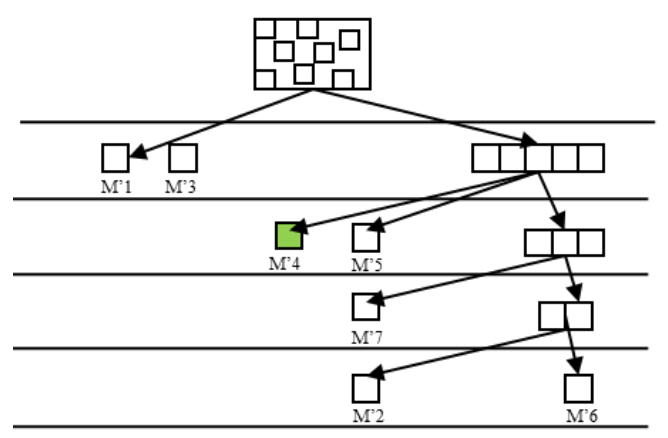

\begin{tabular}{|l|l|l|l|}
\hline From part & Topart & Joining category & Quantity \\
\hline M'1 & M'5 $^{\prime}$ & Insert & 1 \\
\hline M'1 & $M^{\prime} 2$ & Snap fit & 4 \\
\hline M'3 & $M^{\prime} 2$ & Insert & 1 \\
\hline M'4 & $M^{\prime} 7$ & Snap fit & 3 \\
\hline M'4 & M'6 $^{\prime}$ & Screw connection & 4 \\
\hline M'4 & M'5 & Snap fit & 2 \\
\hline M'4 & M'2 & Screw connection & 2 \\
\hline M'5 & $M^{\prime} 7$ & Screw connection & 1 \\
\hline M'5 & M'7 & Snap fit & 4 \\
\hline M'7 & M'6 & Screw connection & 6 \\
\hline M'2 & M'6 & Screw connection & 6 \\
\hline
\end{tabular}

Figure 12: Detail of the $1^{\text {st }}$ potential Product Architecture

Lorsque la séquence de désassemblage est définie ainsi que les éléments de fixation, il est possible de déterminer à l'aide d'un logiciel de simulation les temps de désassemblage et les coûts associés. Ici le calcul des coûts de désassemblage est basé sur un salaire horaire $49 € / \mathrm{h}$ (taxes incluses).

Le

Table 5 répertorie les coûts évités (Recovered Value) lorsqu'un module est remanufacturé, les coûts générés (Loss Value) par l'achat de modules en remplacement des modules recyclés. Par simplification les profits générés par la revente des $\mathrm{pMr}$ aux recycleurs compensent les frais de transport. Lorsque des $\mathrm{pMr}$ se trouvent à la fin de la séquence de désassemblage les coûts sont considérées nul $\left(\mathrm{C}_{\mathrm{dis}}=0\right)$.

Recovered Value : $\mathrm{G}_{\mathrm{pMR}}=\mathrm{P}_{\mathrm{r}}-\mathrm{C}_{\mathrm{dis}}$

Loss Value : $\mathrm{L}_{\mathrm{pMr}}=\mathrm{P}_{\mathrm{r}}+\mathrm{C}_{\mathrm{dis}}$

Avec Pr : prix de revient

Cdis : Coût de désassemblage

Le tableau (

Table 5), synthétise les données relatives à l'architecture 1 (classe des modules, temps de désassemblage/ module, coûts de désassemblage/ module, Prix des modules, coûts évités, coûts générés, impacts évités et impacts générés).

L'architecture potentielle 1 est issue du groupement modulaire 1 dont la stratégie était de récupérer des modules coûteux (plutôt qu'impactant) et de grouper les modules selon leur fiabilité (Figure 10).

Table 5: Economic and environmental data per modules for 1st Product Architecture

\begin{tabular}{|c|c|c|c|c|c|c|c|c|}
\hline Modules & EoL & $t_{\cos }(s)$ & $\mathrm{C}_{\mathrm{s}_{0}}(€)$ & $P_{s}(€)$ & RecoveredValue (€) & Loss Value (E) & $\begin{array}{l}\text { AvoidedImpact } \\
\text { (mPts) }\end{array}$ & $\begin{array}{l}\text { Generated impacts } \\
(\mathrm{mPts})\end{array}$ \\
\hline M1 & $\mathrm{pMr}$ & 7,3 & 0,10 & 5,5 & & 5,60 & & \\
\hline $\mathrm{M} 2$ & $\mathrm{Mr}$ & 1823 & 248 & 75 & 11111 & 9.98 & VIIIS & 253 \\
\hline$M^{2} 3$ & $\mathrm{pMr}$ & $\frac{20.5}{2.5}$ & 0,03 & 22 & & $\frac{9,90}{22,03}$ & 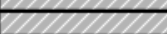 & 320 \\
\hline M4 & $\mathrm{pMR}$ & 76,3 & 1,04 & 32,5 & 31,46 & 780010 & 768 & 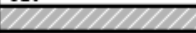 \\
\hline M5 & $\mathrm{pMr}$ & 26,6 & 0.36 & 3.5 & & 3,86 & (1) & 420 \\
\hline M6 & $\mathrm{pMr}$ & 182,3 & 2.48 & 7 & DIIIIIIIIS & 9.48 & DIIIIIIIII & 280 \\
\hline $\mathrm{M}^{2} 7$ & $\mathrm{pMr}$ & $\frac{102,5}{136,5}$ & $\frac{2,40}{1.86}$ & 9 & DIs & 10,86 & $\pi$ & 990 \\
\hline
\end{tabular}

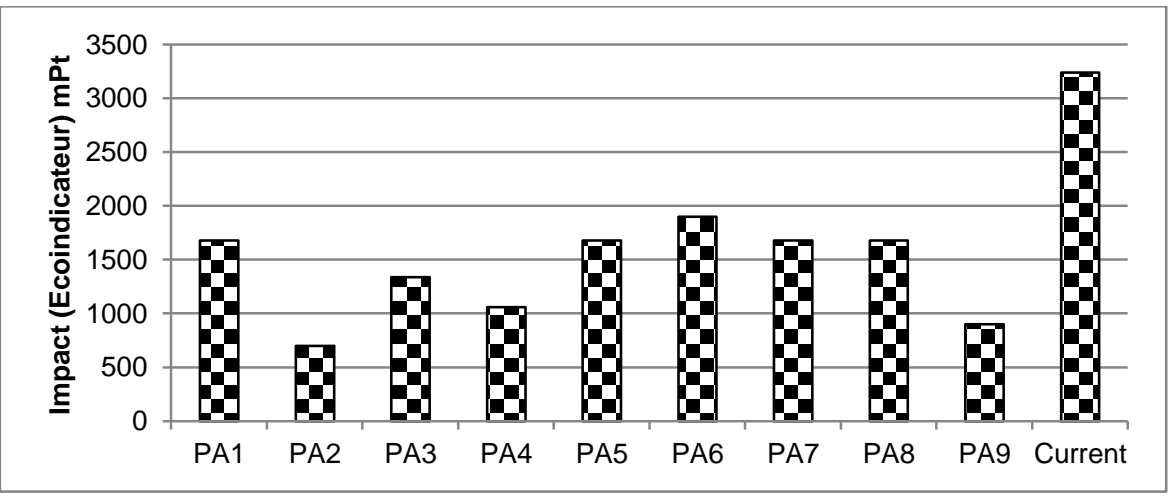

Figure 13 : Environmental Assessment for each PA 
Les 9 Product Architectures générés par l'outil MGE et l'équipe de conception sont comparées avec le produit actuel (Figure 13 et Figure 14).

Le scénario de fin de vie du produit actuel génère un impact de $3200 \mathrm{mPts}$ (Figure 13). Parmi les architectures produit c'est l'architecture produit PA2 qui génère le moins d'impact avec 700mPts soit un gain de 79\% par rapport au scénario actuel, alors que l'architecture produit PA6 génère le plus d'impact avec $1900 \mathrm{mPts}$ soit un gain de $41 \%$ par rapport au scénario actuel.

Concernant les coûts (Figure 14) l'architecture PA2 génère des bénéfices (environ 36 cents d' $€$ ), alors que la solution la plus coûteuse est l'architecture PA6 avec un coût de production estimé à environ $60 €$ soit une économie de $40 \%$ par rapport au scénario actuel.

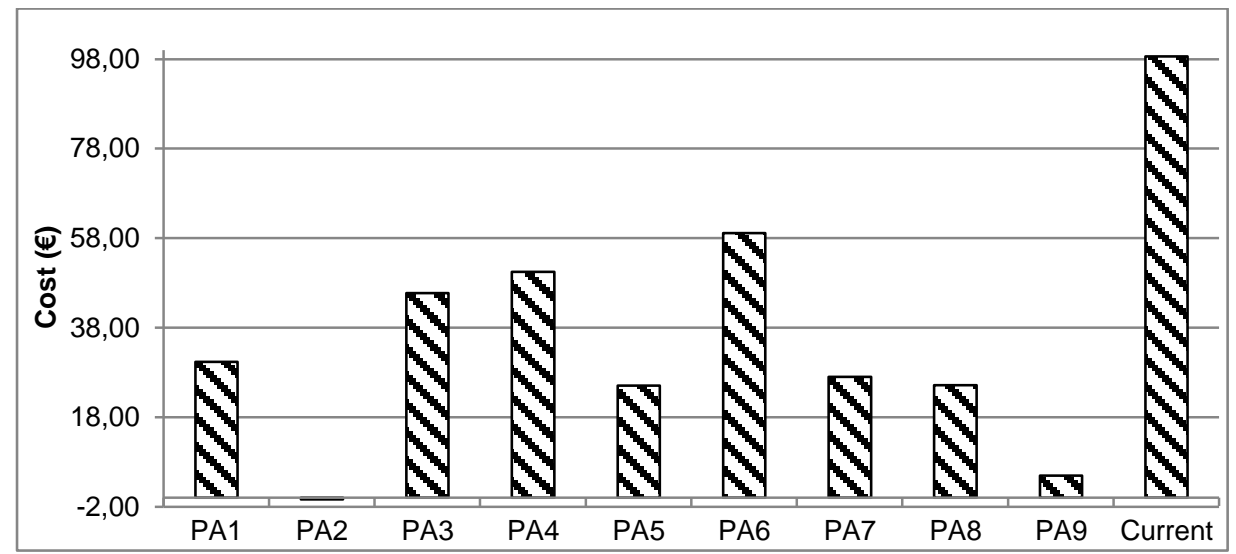

Figure 14 : Economic Assessment for each PA

L'évaluation environnementale privilégie les Architectures Produit constituées d'un grand nombre de modules remanufacturés et impactant et de modules recyclés moins nombreux et peu impactant. L'évaluation économique privilégie les Architectures Produit dont les modules remanufacturés ont une valeur ajoutée plus importante et dont le désassemblage est optimisé. Les deux évaluations aident l'équipe de conception à choisir une Architecture Produits dans un vaste champ de solutions possibles. Dans l'étude A, les architectures 2 et 9 sont les plus performantes sur le plan des coûts et des impacts environnementaux.

\subsection{Etude B : Influence des « Targets » environnemental et économique}

Dans l'étude cas A, la caractérisation des modules (pMR, pMr et pMU) s'appuie sur des « Seuils » ou curseurs économique et environnemental définie par le couple $\left(\mathrm{T}^{\mathrm{pc}} ; \mathrm{T}^{\mathrm{ei}}\right)$. Le choix de ces seuils influe grandement sur le nombre de solutions de regroupement.

Intuitivement trois composants constitutifs de la machine à café sont susceptibles d'être remanufacturés car coûteux et/ ou impactant : l'infuseur, la pompe et la chaudière (Figure 7). Dans cette stratégie l'exploration du champ des possibilités se limite à une architecture figée où le concepteur définit un scénario de fin de vie adapté aux différents composants et modules. Cette approche intuitive, ne remettant pas suffisamment en cause l'architecture du produit, ne peut aboutir qu'à de simples optimisations locales sur le choix des matériaux et des fixations des modules.

L'étude B vise à regarder l'incidence d'une augmentation/ ou diminution d'un des seuils Tpc/ Tei sur la définition des frontières des modules du produit, dont une représentation schématique est présentée Figure 7 .

L'analyse environnementale et économique du produit actuel montre que la pompe est un composant intéressant à remanufacturer. Afin de mesurer l'influence des seuils sur la caractérisation et la définition des frontières du module Pompe, nous avons fait varier $\mathrm{T}^{\mathrm{pc}}$ indépendamment en fixant $\mathrm{T}^{\mathrm{e}}$.

Le couple de référence est $(5,10)$ (correspondant au scénario 0 ), la classification des modules et les architectures produit découlant de ce couple sont présenté dans l'étude A. 3 scénarios sont définis : S1 avec $\mathrm{T}^{\mathrm{pc}}=5, \mathrm{~S} 2$ avec $\mathrm{T}^{\mathrm{pc}}=15$ et $\mathrm{S} 3$ avec $\mathrm{T}^{\mathrm{pc}}=20$. $\mathrm{T}^{\mathrm{ei}}$ étant fixé à 5.

Le tableau 5 (Table 6) présente l'évolution de la caractérisation des modules. En augmentant le seuil $\mathrm{T}^{\mathrm{pc}}$ la part de modules pMR diminue tandis que la part de modules pMU et pMr augmente. La présence de module pMU permet des regroupements soit avec de pMR soit avec de pMr mais aussi autorise le concepteur à splitter le module pour réassocier un de ces sous-modules à un autre module de la même classe (tout cela selon le processus itératif défini précédemment). Contrairement à d'autres méthodes de « Design for Modularity » MGE ne converge pas vers une solution unique optimisé, au contraire MGE cherche à explorer le maximum de combinaisons possibles. 
Table 6 : Influence des "seuils" Coûts et Impact Environnemental sur la catégorisation des modules
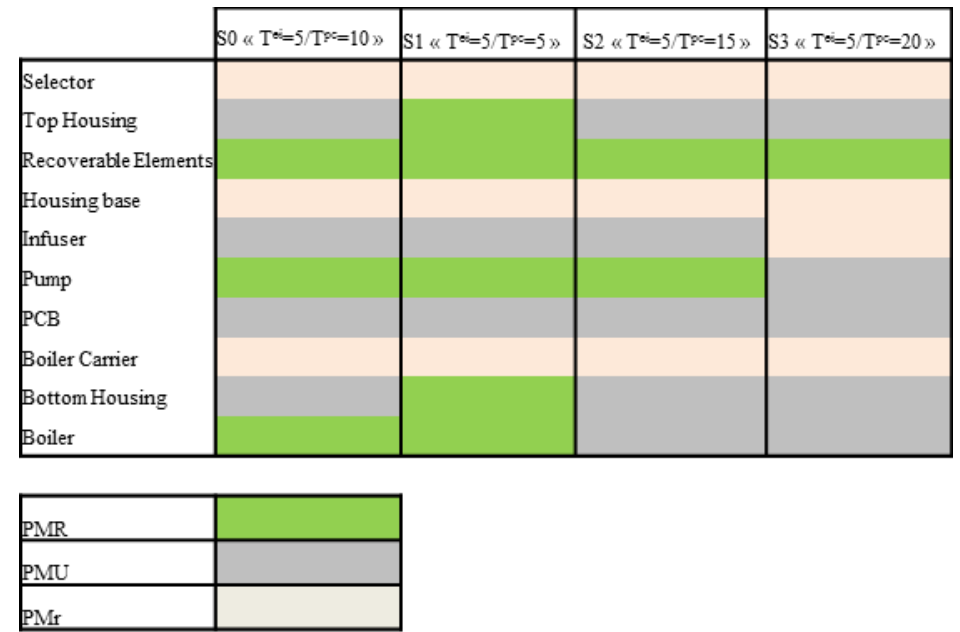

Reprenons l'exemple de la pompe, pour les 3 couples $(\mathrm{Tpc}=5 / \mathrm{Tei}=5),(\mathrm{Tpc}=15 / \mathrm{Tei}=5)$ et $(\mathrm{Tpc}=20 / \mathrm{Tei}=5)$, le module pompe est caractérisé comme pMR. Pour ces trois couples de seuils économique et environnemental MGE propose les regroupements (Figure 15).

Lorsque l'un des deux seuils augmente, la caractéristique de la pompe passe de pMR à PMU, alors que la majorité des autres modules passe de pMU à pMr. MGE propose alors de splitter la pompe en deux modules Pompe' (pMR) et pompe"' (pMr), ensuite par itération chacun des deux modules sera éventuellement regrouper avec respectivement des modules pMR et pMr. C'est le cas dans le scénario 4 avec le groupement d'une partie de la pompe avec le carter inférieur au sein d'un module recyclable.

Par contre lorsque l'un des deux seuils diminue, la caractéristique de la pompe reste pMR mais certains modules passent de pMr ou pMU à respectivement pMU ou pMR. MGE va alors permettre des regroupements plus importants. Par exemple, pour le scénario 1 la pompe peut être groupée avec l'infuseur et le carter inférieur ou avec le PCB et module inférieur au sein d'un module remanufacturable.

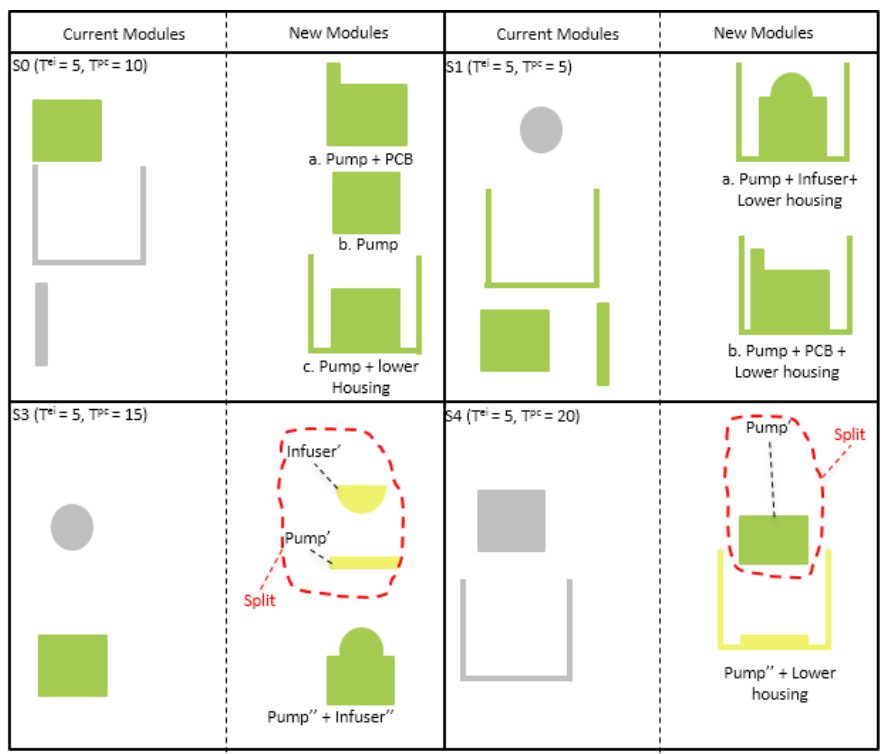

PMR

pMU

$\mathrm{pM}$

Figure 15 : Incidence des seuils éco-environnementaux sur la granularité du module pompe

\subsection{Etude C : Sensibilité sur les valeurs MTBF et VLT}

Le regroupement modulaire (MGs) est basé sur la connaissance des données de fiabilité (MTBF) et d'obsolescence (VLT). La connaissance précise de ces valeurs est délicate car dépendantes de 
fournisseurs externes. De plus MGE à besoin de données prospectives sur la fiabilité/ obsolescence de composants améliorés.

Pour cette étude sensibilité nous avons faire varier les données de fiabilité et d'obsolescence de $\square=5,10$, $15,20 \%$.

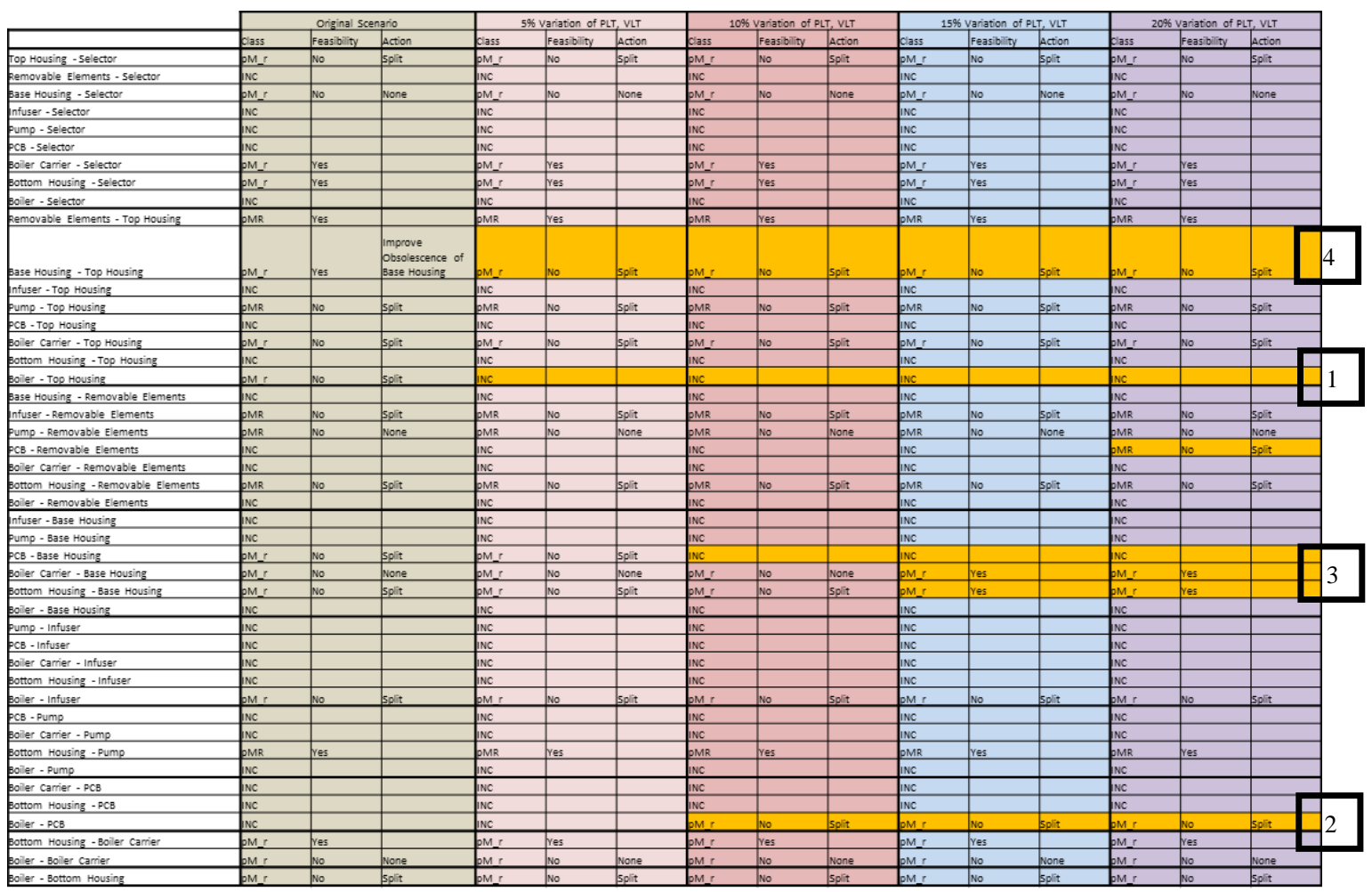

Figure 16 : Evolution des MGs en fonction des données MTBF et VLT

Sur les 47 MGs évalués par l'outil MGE, les modifications engendrées par une variation des données de fiabilité et d'obsolescence sont limitées entre 4 et 15\% par rapport au scénario d'origine (Table 7).

- Des regroupements entre pMR deviennent incompatible (cas 1 sur la Figure 16) car si MTBF et/ ou VLT varie, la validation des modules pMR est affecté. Par exemple si au départ on a un regroupement $\mathrm{pMR}_{\mathrm{a}} / \mathrm{pMR}$ et que diminue alors $\mathrm{pMR}_{\mathrm{b}}$ devient $\mathrm{pMr}_{\mathrm{b}}$ avec comme conséquence de rendre $\mathrm{pMR}_{\mathrm{a}}$ et $\mathrm{pMr}_{\mathrm{b}}$ incompatible.

- Inversement des groupements incompatibles peuvent devenir potentiellement compatibles (cas 2 sur la Figure 16) du fait d'une modification de MTBF et ou VLT. Par exemple, $\mathrm{pMR}_{\mathrm{a}} / \mathrm{pMr}_{\mathrm{b}}$ sont incompatibles car de classes différentes mais si MTBF et ou VLT évoluent, $\mathrm{pMR}_{\mathrm{a}}$ devient $\mathrm{pMr}_{\mathrm{a}}$ et le groupement $\mathrm{pMr}_{\mathrm{a}} / \mathrm{pMr}_{\mathrm{b}}$ devient potentiellement possible.

- Des groupements non possibles entre 2 modules de mêmes classes, à cause d'une affinité insuffisante, peuvent devenir possible si MTBF et/ou VLT sont modifiées (cas 3 sur la Figure 16).

- Des groupements possibles entre 2 modules de mêmes classes, grâce à une affinité suffisante, peuvent devenir impossible si MTBF et/ou VLT sont modifiées (cas 4 sur la Figure 16).

MGE ne nécessite pas d'avoir une précision importante des données d'obsolescence et de fiabilité pour générer des groupements modulaires. Une approximation de ces données de l'ordre de 5 à $10 \%$ par l'équipe de conception est suffisante. Ainsi l'équipe de conception peut concentrer ces efforts sur la phase de définition des liaisons des architectures potentielles.

Table 7 : Influence de l'incertitude des données de MTBF et VLT sur le MGs

Overview of modifications 


\begin{tabular}{|c|c|c|c|c|c|c|c|c|c|c|}
\hline$\Delta$ & $\begin{array}{l}\text { Number of modification } \\
\text { Compared with original } \\
\text { scenario }\end{array}$ & $\%$ modif & $\mathrm{pMX}->\mathrm{INC}$ & $\%$ & INC->pMX & $\%$ & No->Yes & $\%$ & yes->No & $\%$ \\
\hline $5 \%$ & $2(47)$ & 4,26 & $1(20)$ & 5 & $0(27)$ & 0 & $0(14)$ & 0 & $1(6)$ & 16,67 \\
\hline $10 \%$ & $4(47)$ & 8,51 & $2(20)$ & 10 & $2(27)$ & 7,41 & $0(14)$ & 0 & $1(6)$ & 16,67 \\
\hline $15 \%$ & $6(47)$ & 12,77 & $2(20)$ & 10 & $1(27)$ & 3,70 & $2(14)$ & 14,29 & $1(6)$ & 16,67 \\
\hline $20 \%$ & $7(47)$ & 14,89 & $2(20)$ & 10 & $2(27)$ & 7,41 & $2(14)$ & 14,29 & $1(6)$ & 16,67 \\
\hline
\end{tabular}

\section{Conclusion}

L'approche suggérée dans cet article et matérialisée par l'outil Modular Grouping Explorer (MGE) permet de prédéfinir des modules dans les phases amont du processus de conception. Ces modules sont établis afin d'optimiser la fin de vie, en regroupant les modules/ composants selon différentes caractéristiques de leur cycle de vie. Les groupements modulaires prennent en compte les caractéristiques physiques (fiabilité) des composants ainsi que leur obsolescence (VLT) à travers le cycle de vie. Cette approche a été mise en œuvre sur une machine expresso dans le but de générer des architectures modulaires potentielles remanufacturables. MGE permet un spectre d'exploration large de solutions selon des critères économiques et environnementales.

Cet outil fait partie d'une méthodologie plus complète intégrant la définition de cycle d'Upgrade (Pialot, 2011), de structure de reverse supply chain (El Korchi, 2010) et permettant des activités de remanufacturing économiquement viable tout en prenant compte des considérations économiques et sociales.

Les avantages de MGE par rapport à d'autres outils similaires sont :

- L'outil MGE propose une approche qui va au-delà de la simple optimisation des scénarios de fin de vie sans toutefois remettre en cause l'architecture complète du produit, les modules et les composants.

- MGE permet une meilleure exploration du champ du possible en conception par la possibilité qui est offerte au concepteur de redéfinir les frontières initiales des modules (split or group). En élargissant le faisceau (spectre) de solutions on évite d'exclure à priori certaines solutions innovantes.

- MGE est un outil qui sollicite l'intelligence des acteurs de la conception du produit contrairement à certains outils systématiques. L'outil MGE se contente alors de jouer un rôle de support du processus exploratoire réalisé par le collectif de conception.

Dans l'avenir, cet outil « Modular Grouping Explorer » (MGE) pourrait être enrichi de la façon suivante :

- Prendre en compte l'impact environnemental à l'utilisation en plus de l'extraction/ fabrication comme clé de catégorisation des modules pMR.

- Générer un graphe de démontage initial puis final. Cela permettrait de comparer l'état initial de l'architecture produit avec les architectures proposées par l'outil et ainsi de justifier les gains d'une nouvelle architecture.

- Génération par l'outil, en parallèle ou sur le graphe de démontage, des nouveaux coûts de désassemblage et impact environnementaux. Puis affichage des points d'amélioration et de dégradation par rapport à la performance du produit initial.

- Amélioration de l'ergonomie de l'outil.

- Mise en place d'une aide en ligne pédagogique pour l'utilisateur.

\section{Acknowledgements}

The authors would like to thank ADEME and CETIM foundation for their financial support in the framework of MacPMR research program.

\section{References}

Amezquita T, Hammond R, Salazar M, Bras B. Characterising the remanufacturing of engineering systems. In: Proceedings of 1995 ASME advances in design automatization conference. Boston, Massachusetts DE, vol. 82; September, 1995.

Bras B, Hammond R., Towards design for remanufacturing : metrics for assessing remanufacturing. In: Flapper SD, de Ron AJ, editors. 1996.

Desai, A., \& Mital, A., (2003), Evaluation of disassemblability to enable design for disassembly in mass production. International Journal of Industrial Ergonomics, pp.32, 265-281. 
Duflou, J.R., Seliger, G., Kara, S., Umeda, Y., Ometto, A., \& Willems, B., (2008), Efficiency and feasibility of product disassembly: A case-based study. Paper presented at the CIRP Annals - Manufacturing Technology.

El Korchi, A., (2010), Conditions stratégiques d'émergence d'une reverse supply chain fondée sur le remanufacturing chez le fabricant d'origine, The 8th International Conference on Logistics and SCM Research, Bordeaux, 2010.

Erens, F. and Verhulst, K., (1997), Architectures for product families. Computers in Industry, 33, 165-178.

Europa. Directive 2000/53/EC of the European Parliament and of the Council of 18 September 2000 on end-of life vehicles (ELV). http://europa.eu.int , 2000.

Europa. Directive 2002/96/EC of the European Parliament on waste electrical and electronic equipment (WEEE). Official Journal of the European Union, L37/24, pp. 1-15, http://europa.eu.int, 2003.

Fixson, S.K., (2005), Product architecture assessment: a tool to link product, process, and supply chain design decisions. Journal of Operations Management. v23. 345-369.

Gehin A., (2008), Développement d'une Méthodologie de Conception de Produits Durables. PhD Thesis INPG Génie Industriel.

Giudice, G. (2010) Disassembly depth distribution for ease of service: a rule-based approach. Journal of Engineering Design, Volume 21, Issue 4, pp. 375-411.

Gungor A, Gupta S M., (1997), An evaluation methodology for disassembly process. Computers \&Industrial Engineering, 33, pp.329-332.

ljomah, W.L., (2002), A model-based definition of the generic remanufacturing business process. University of Plymouth.

ljomah, W.L., McMahon, C.A., Hammond, G.P., \& Newman, S.T., (2007), Development of design for remanufacturing guidelines to support sustainable manufacturing. Robotics and Computer-Integrated Manufacturing, 23, pp.712719.

Ishii, K., Eubanks, C.F., Marks, M. (1992) Evaluation Methodology for Post-Manufacturing Issues in Life-cycle Design, Concurrent Engineering: Research and Applications. Vol.1, pp. 61-68.

Kara S, Pornprasitpol P, H. Kaebernick H., (2006), Selective Disassembly Sequencing: A Methodology for the Disassembly of End-of-Life Products. Annals of CIRP, 55, pp1-4.

Kerr W., (1999), Remanufacturing and eco-efficiency: A case study of photocopier remanufacturing at Fuji Xerox Australia. International Institute for Industrial Environmental Economics: Lund University, Sweden.

Kimura F, Kato S, Hata T, Masuda T., (2001), Product modularization for parts Reuse in inverse manufacturing. Annals of CIRP, 50, pp.89-92.

Kiritsis, D., Bufardi, A., and Xirouchakis, P., (2003) Multi-Criteria Decision Aid for Product End of Life Options Selection. Proceedings of the 2003 IEEE International Symposium on Electronics \& the Environment, Boston, USA, 2003.

Kondoh, S., Umeda, S., \& Yoshikawa H., (1998), Development of Upgradable Cellular Machines for Environmentally Conscious Products. Annals of CIRP, 47(1).

Lambert A. J. D., (2002), Determining Optimum Disassembly Sequences In Electronic Equipment. Computers \&Industrial Engineering, 43, pp.553-575.

Lee, S.G. Lye, S.W. and Khoo, M.K. (2001) A multi-objective methodology for evaluating product end-of-life options and disassembly, The International Journal of Advanced Manufacturing Technology, 18 (2001), pp. 148-156.

Lund, R.T., (1996), The Remanufacturing Industry: Hidden Giant. Boston. Boston: Argonne National Laboratory study

Mont, O., Dalhammar, C., Jacobsson, N., (2006), A new business model for baby prams based on leasing and product remanufacturing. Journal of Cleaner Production, 14(17), pp.1509-1518.

Pialot, O., Millet D., Tchertchian, N., (2010), Definition of potential upgrade scenario in early design phases of remanufacturable system. Proceedings of IDMME - Virtual Concept Bordeaux, 2010.

Remery, M., Mascle, C., Agard, B. (2012) A new method for evaluating the best product end-of-life strategy during the early design phase. Journal of Engineering Design, Volume 23, Issue 6, pp. 419-441.

Rose, C. M., Beiter, K. A., Ishii, K. "Determining End-Of-Life Strategies As A Part Of Product Definition." International Symposium on Electronics And The Environment, IEEE 1999

Rose, C. M., Ishii, K. and Stevels, A., "Influencing Design to Improve Product End-Of-Life Stage", Research in Engineering Design, Vol. 13, No. 2, 83-93, 2002.

Rose, C.M., Ishii, K., Masui, K. (1998), "How product characteristics determine end-of-life strategies", Proceedings of the 1998 IEEE International Symposium on Electronics and the Environment, ISEE-1998, Oak Brook, IL, USA, pp.322-7.

Rose, C.M., Stevels, A. and Ishii, K., 2000, "A new approach to End-of-Life Design Advisor (ELDA)," Proceedings of the 2000 IEEE International Symposium on Electronics and the Environment, IEEE, pp. 99-104.

Seliger G, Zettl M., (2008), Modularization as an enabler for cycle economy. Annals of CIRP - Manufacturing Technology, 57, pp.133- 136.

Steinhilper R., (1998), Remanufacturing - The Ultimate Form of Recycling: Fraunhofer IRB Verlag, Stuttgart.

Sundin E., (2004), Product and Process Design for Successful Remanufacturing. Department of Mechanical Engineering. Linköping, Sweden: Linköping University.

Sundin, E., 2001. Product properties essential for remanufacturing. In: Proceedings to 8th international seminar on Life Cycle Engineering (LCE). Sponsored by International Institution for Production Engineering Research (CIRP), June 18-20, Varna, Bulgaria, pp. 171-179.

Takeuchi, S. and Saitou, K. (2006) Design for optimal end-of-life scenario via product-embedded disassembly, Proceedings of the ASME Design Engineering Technical Conferences, Philadelphia, Pennsylvania, September 10-13, DETC2006-99475.

Tchertchian, N., \& Liang, H., Millet, D., (2009), The Influence of the Multiple Life Cycles on the Environmental Impact of a Product. Proc. ICED 2009 17th Int. Conf. Engineering Design.

Tchertchian, N., Millet D., El Korchi A., (2010), A method helping to define eco-innovative systems (product architecture + RSC structure + scenario usage). 17th CIRP International Conference on Life Cycle Engineering, Hefei. 
Tomiyama, T., Sakao, T., \& Umeda, Y., (2005), The Post Mass Production Paradigm, Knowledge Intensive Engineering, and Soft Machine. In International Conference on Life Cycle Modelling for Innovative Products and Processes. Berlin.

Tonnelier, P., Millet D., Richir S. and Lecoq M. (2005) Is it possible to evaluate the recovery potential earlier in the design process : proposal of a qualitative evaluation tool. Journal of Engineering Design, Volume 16, Issue 3, pp. 297-309.

Tseng H.-E., Chang C.-C., Li J.-D., Modular design to support green life-cycle engineering. Expert Systems with Applications, 34:25, 24-37, 2008.

Ulrich, K. T., (1995), The role of product architecture in the manufacturing firm, Research Policy. 24: 419-440.

Umeda Y, Fukushige S, Tonoike K, and Kondoh S., (2008), Product modularity for life cycle design. CIRP Annals Manufacturing Technology, 57, pp.13-16.

Van der Laan, E., Salomon, M., (1997), Production planning and inventory control with remanufacturing and disposal. European Journal Of Operational Research, 102, pp.264-278.

Wang, W.Y. and Tseng, M.M. (2009) Life Cycle Commonality: As a Systematic Approach to Achieve Product Design for Sustainability, Proceedings of DET2009 - 6th International Conference on Digital Enterprise Technology, Hong Kong, pp. 289-303, 14-16 December 2009.

William, J. and Shu L., Analysis of remanufacturer waste streams across product sectors. CIRP Annals 2001; 50(1):101e4, 2001.

Xing, K., L. Luong, and K. Abhary (2003) "IREDA: An Integrated Methodology for Product Recyclability and End-of-Life Design", Journal of Sustainable Product Design, Vol.3, Issue 4, pp 149 172, Springer.

Zuidwijk R, \& Krikke H., (2007), Strategic Response to EEE Returns: Product Eco-Design Or New Recovery Process? European Journal of Operational Research, 191, pp.1206-1222.

Zwolinski, P., Brissaud, D. (2008) Remanufacturing strategies to support product design and redesign. Journal of Engineering Design, Volume 19, Issue 4, pp. 321-335. 